\title{
Pairwise-stability and Nash equilibria in network formation
}

\author{
Antoni Calvó-Armengol • Rahmi İlkılıç
}

Accepted: 14 August 2008 / Published online: 18 September 2008

(C) The Author(s) 2008. This article is published with open access at Springerlink.com

\begin{abstract}
Suppose that individual payoffs depend on the network connecting them. Consider the following simultaneous move game of network formation: players announce independently the links they wish to form, and links are formed only under mutual consent. We provide necessary and sufficient conditions on the network link marginal payoffs such that the set of pairwise stable, pairwise-Nash and proper equilibrium networks coincide, where pairwise stable networks are robust to one-link deviations, while pairwise-Nash networks are robust to one-link creation but multilink severance. Under these conditions, proper equilibria in pure strategies are fully characterized by one-link deviation checks.
\end{abstract}

Keywords Network formation · Pairwise-stability $\cdot$ Proper equilibrium

JEL Classification $\quad \mathrm{C} 62 \cdot \mathrm{C} 72 \cdot \mathrm{D} 85 \cdot \mathrm{L} 14$

\begin{abstract}
Antoni Calvó-Armengol heart breakingly passed away on 3 November 2007. His memory will forever remain radiant and warm, like he always was.

We thank William Thomson, an associate editor and two anonymous referees for their suggestions that led to substantial improvements. We also thank Sjaak Hurkens, Bettina Klaus, Jordi Massó and Giovanni Neglia for helpful conversations. The first author gratefully acknowledges the financial support from the Spanish Ministry of Education and FEDER through grant SEJ2005-01481/ECON, the Fundación BBVA and the Barcelona Economics Program of XREA. The second author is grateful to the Netherlands Organization for Scientific Research (NWO) for its support under grant VIDI-452-06-013.
\end{abstract}

A. Calvó-Armengol

Department of Economics, ICREA, Universitat Autònoma de Barcelona, UAB, Edifici B, 08193 Bellaterra (Barcelona), Spain

R. İlkılıç ( $\varangle)$

Department of Economics, Universiteit Maastricht, PO Box 616, 6200 MD Maastricht, The Netherlands

e-mail: r.ilkilic@algec.unimaas.nl 


\section{Introduction}

When individual payoffs depend on an underlying network of bilateral links, selfinterested players may want to manipulate the network structure to their advantage. A model of network formation needs to specify how players set up links with each other, together with a network equilibrium concept compatible with this process. In recent years, different network formation procedures and network stability concepts have been proposed. This paper aims at building a bridge between these concepts. We relate pairwise-stability, a prominent network equilibrium concept due to Jackson and Wolinsky (1996), to proper equilibrium, a non-cooperative refinement due to Myerson (1978).

By definition, pairwise stable networks are robust to one-link deviations. Such deviations are either promoted by single players in isolation (in the case of linkcutting), or at the coordinated initiative of pairs of players (in the case of link-creation). Pairwise-stability is a weak equilibrium notion, often thought of as a necessary condition for network stability, ${ }^{1}$ and it is independent from any procedure of network formation. It was introduced initially to highlight the inherent tension between stability and efficiency in a network context. Pairwise-stability is also extensively used for positive purposes due to its computational (relative) simplicity, and to its ability to generate sharp predictions in many contexts. ${ }^{2}$

Myerson (1991) proposes a normal form game of network formation. In this game, players simultaneously announce all the links they wish to form. The resulting network is formed by the mutually announced links. This game is simple and intuitive. But, given that link creation requires the mutual consent of the two involved parties, a coordination problem arises. As such, the game displays a multiplicity of Nash equilibria, and very different network geometries can arise. ${ }^{3}$

To be precise, the coordination problem in the Myerson game is a consequence of the multidimensional strategy space (players can announce any combination of links they wish), combined with the requirement of mutual consent. If players are allowed to coordinate bilaterally, instead, no mutually beneficial link is left aside. We call pairwise-Nash networks the Nash equilibrium outcomes that fulfill this added (coalitional move) requirement. ${ }^{4}$

\footnotetext{
1 Most likely, any equilibrium notion should, at least, check for one-link deviations.

2 See Jackson (2004) for an exhaustive survey on these issues.

3 For undirected networks, where link creation requires mutual consent, the empty network is always a Nash equilibrium outcome (when nobody announces any link). Instead, for directed networks, where arrow-pointing links need not be reciprocated, a standard Nash equilibrium analysis can narrow down reasonably the geometry of endogenous networks. Bala and Goyal (2000), for instance, offer an analysis of Nash directed networks for the Myerson game where payoffs correspond to (variations of) the connections' model.

4 The set of pairwise-Nash networks is thus at the intersection of the set of Nash equilibrium outcomes and the set of pairwise stable networks. See Goyal and Joshi (2006), Calvó-Armengol (2004) and Bloch and Jackson $(2006,2007)$ for definitions and applications of pairwise-Nash networks. See also Dutta and Mutuswami (1997) and Jackson and van den Nouweland (2005) for alternatives to pairwise stability and Nash equilibrium that allow for coalitional moves. Dutta et al. (1998) contains an exhaustive analysis of network formation in a cooperative set up.
} 
Our first result is that pairwise-stability and pairwise-Nash equilibrium are equivalent when a simple condition on link marginal returns holds. This condition, $\alpha$-submodularity, involves comparing joint returns from a set of existing links with the sum of the marginal returns from each of them. Pairwise-Nash networks are robust to multi-link severance and single-link creation, a stronger requirement than the singlelink robustness check of pairwise stability. Under $\alpha$-submodularity, robustness to unilateral or multilateral link severance turn out to be equivalent, and the result follows. We show that many existing models in the literature fulfill this condition, including the connections model, the coauthor model, and models of information transmission on the network.

Pairwise-Nash equilibrium lies at the crossroad of cooperative and non-cooperative games. In particular, the process through which pairs of players coordinate their link announcements is left unspecified. To avoid such ad hoc coalitional moves, we focus on proper equilibrium, a Nash refinement due to Myerson (1978). In a proper equilibrium, players best respond to perturbations of their opponents' strategies, where perturbations are ordered so that more costly mistakes are made with smaller probability. ${ }^{5}$

We provide conditions on link marginal payoffs such that pairwise-Nash equilibria and proper equilibria coincide.

We first show that every pairwise-Nash network is also proper under two conditions. The first one, weak link-responsiveness, states that the returns to any group of current links are never zero. This is a mild requirement. Network payoffs with exogenous parameters are generically weakly link-responsive. The second condition, $\beta$-strong supermodularity, compares the joint returns from a set of new links to the sum of the marginal returns from each of them. In fact, $\beta$-supermodularity is only required for a particular set of links. We impose this condition for the links absent from the network such that one player gains from adding this new link whereas the other loses. This condition thus trivially holds in the complete network, where all possible links are already formed, and in networks where neither player consents in creating an absent link. More generally, this property holds for a variety of models, including the connections model and the model of information transmission. ${ }^{6}$

We also show that every proper equilibrium network is a pairwise-Nash network when payoffs are strongly link-responsive. Strong link-responsiveness differs from the weak version outlined above. Given a network it requires that no player is indifferent to a change in his set of direct links, whether due to formation, link removal, or a combination of both. We impose this condition only on Nash equilibrium networks, not on the set of all possible networks. We also provide an example with strongly link-responsive payoffs for which a perfect equilibrium network is not pairwise-Nash, thus the need to resort to properness.

The paper is organized as follows. Section 2 describes networks, payoffs and defines $\alpha$-submodularity and $\beta$-strong supermodularity. Section 3 introduces network forma-

\footnotetext{
5 Ordered mistakes differ by, at least, one order of magnitude. This hierarchy of mistakes distinguishes properness from trembling-hand perfection.

6 In particular, this shows that $\alpha$-convexty and $\beta$-strong supermodularity are perfectly compatible with one another. Recall that $\alpha$-submodularity refers to current links, while $\beta$-supermodularity refers to new links.
} 
tion games and stability concepts. Section 4 contains the main results, and Sect. 5 develops some examples. We discuss possible extensions in Sect. 6. The proofs of the main results are gathered in Sect. 7. An Appendix gathers extra material.

\section{Network payoffs}

Networks Let $N=\{1, \ldots, n\}$ be the set of players who may be involved in a network. A network ${ }^{7} g$ is a list of pairs of players who are linked to each other. We denote the link between two players $i$ and $j$ by $i j$, so $i j \in g$ indicates that $i$ and $j$ are linked in the network. Let $g^{N}$ be the set of all subsets of $N$ of size 2. The network $g^{N}$ is referred to as the complete network. The set $\mathcal{G}=\left\{g \subseteq g^{N}\right\}$ denotes the set of all possible networks on $N$. The set of $i$ 's direct links in $g$ is $L_{i}(g)=\{j k \in g: j=i$ or $k=i\}$ and $L_{i}\left(g^{N} \backslash g\right)=\{i j: j \neq i$ and $i j \notin g\}$ is the set of $i$ 's direct links not in $g$. That is, $i j \notin g$ is equivalent to $i j \in L_{i}\left(g^{N} \backslash g\right)$.

Let $g+i j$ denote the network obtained by adding the link $i j$ to the network $g$ and $g-i j$ denote the network obtained by deleting the link $i j$ from the network $g$. More generally, for every collection of links $\ell \subseteq L_{i}(g), g-\ell$ is the network obtained from $g$ by eliminating all the links in $\ell$, while for every collection of links $\ell \subseteq L_{i}\left(g^{N} \backslash g\right)$, $g+\ell$ is the network obtained from $g$ by adding all the links in $\ell$.

Network payoffs A network payoff function is a mapping $u: \mathcal{G} \rightarrow \mathbb{R}^{N}$ that assigns to each network $g$ a payoff $u_{i}(g)$ for each player $i \in N$.

Link marginal payoffs $\quad$ Let $g \in \mathcal{G}$. For all $i, j \in N$ such that $i j \in g$ :

$$
m u_{i}(g, i j)=u_{i}(g)-u_{i}(g-i j)
$$

is the marginal payoff to $i$ from the link $i j$ in $g$. More generally, let $\ell \subseteq L_{i}(g)$. The joint value to $i$ of $\ell$ is:

$$
m u_{i}(g, \ell)=u_{i}(g)-u_{i}(g-\ell) .
$$

Consider now some link $i j \notin g$. Then, $m u_{i}(g+i j, i j)$ is the marginal payoff accruing to $i$ from the link $i j$ being added to $g$. More generally, consider a collection of $i$ 's links absent from $g, \ell \subseteq L_{i}\left(g^{N} \backslash g\right)$. The joint value to $i$ of these new links is $m u_{i}(g+\ell, \ell)=u_{i}(g+\ell)-u_{i}(g)$.

\section{$\alpha$-submodularity in own current links}

Definition 1 Let $\alpha \geq 0$. The network payoff function $u$ is $\alpha$-submodular in own current links on $\mathcal{A} \subseteq \mathcal{G}$ if and only if:

$$
m u_{i}(g, \ell) \geq \alpha \sum_{i j \in \ell} m u_{i}(g, i j),
$$

for all $g \in \mathcal{A}, i \in N$ and $\ell \subseteq L_{i}(g)$.

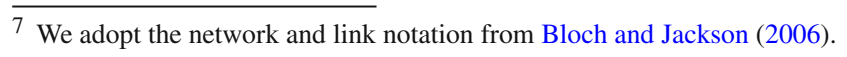


The condition for $\alpha$-submodularity states that the joint returns from a group of links already in the network is higher than the sum of the marginal returns of each single link, scaled by $\alpha$. The case $\alpha=1$ corresponds to submodularity. ${ }^{8}$

Note that $\alpha$-submodularity is a property that applies to marginal returns from existing links only. The condition does not rule out that joint returns from groups of links are less than the sum of marginal returns of single links, as long as the former is non-negative. It is less restrictive than submodularity.

If the single marginal link payoffs from existing links on a network $g$ are negative, then it is possible to find an $\alpha>0$ that satisfies (1). The condition is restrictive when the single link marginal payoffs are non-negative. In that case, $\alpha$-submodularity boils down to a condition on the sign of joint returns from groups of links.

Proposition 1 Suppose $g$ is a network such that for all $i j \in g, m u_{i}(g, i j) \geq 0$. The network payoff function $u$ is $\alpha$-submodular in own current links on $g$ if and only if for all $i \in N$ and all $\ell \subseteq L_{i}(g), m u_{i}(g, \ell) \geq 0 .{ }^{9}$

\section{$\beta$-strong supermodularity in own new links}

Definition 2 Let $u$ a network payoff function, $g \in \mathcal{G}$ a network, $i \in N$ a player, and $\Lambda \subseteq L_{i}\left(g^{N} \backslash g\right)$ a collection of $i$ 's direct links not in $g$. Let $\beta>0$. Then, $u_{i}$ is $\beta$-strongly supermodular in own new links on $\Lambda$ at $g$ if and only if $\Lambda=\varnothing$, or $\Lambda \neq \varnothing$ and:

$$
u_{i}\left(g+\ell-\ell^{\prime}\right)-u_{i}(g) \leq \beta \sum_{i j \in \ell} m u_{i}(g+i j, i j)
$$

for all $\ell \subseteq \Lambda, \ell \neq \varnothing$ and $\ell^{\prime} \subseteq L_{i}(g){ }^{10}$

Let first $\ell^{\prime}=\emptyset$. Then, the left-hand side of (2) is $m u_{i}(g+\ell, \ell)$, that is, the returns to player $i$ in the network $g$ of adding the links $\ell$. Condition (2) states that the joint value from adding these links is lower than the sum of the marginal returns from adding each single such link, scaled by $\beta$. The case $\beta=1$ corresponds to supermodularity, the dual of submodularity defined above.

Let now $\ell^{\prime} \neq \varnothing$. Then, (2) compares the sum of single-link marginal values for links not in the network yet to the joint returns of adding these links at once, while simultaneously cutting any possible subset of $i$ 's current links.

If the single marginal link payoffs from links in $\Lambda$ are positive, then it is possible to find a $\beta>0$ that satisfies (2). The condition is restrictive when the single link marginal payoffs from those links are negative. In that case $\beta$-supermodularity boils down to a condition on the sign of joint returns from adding new links while cutting existing links.

\footnotetext{
8 See, e.g., Bloch and Jackson (2006) for a similar definition. Actually, we borrow the notation for marginal link payoffs from them. $\alpha$-submodularity is closely related with payoff convexity defined in Gilles and Sarangi (2005). Their condition follows directly from $\alpha$-submodularity.

9 The result follows from the definition.

10 Note that $u_{i}\left(g+\ell-\ell^{\prime}\right)-u_{i}(g)=m u_{i}(g+\ell, \ell)-m u_{i}\left(g, \ell^{\prime}\right)$.
} 
In (2) the comparison between payoffs is not quantitative, but only signwise. $\beta$-supermodularity does not rule out that joint returns from groups of links are more than the sum of marginal returns of single links, as long as the former has the same sign as the latter. It is less restrictive than supermodularity.

\section{Network equilibrium}

In what follows, we first define pairwise-stability, due to Jackson and Wolinsky (1996), and often interpreted as a necessary condition for network equilibrium. ${ }^{11}$ We then formulate a simultaneous move game of network formation due to Myerson (1991). This game is simple and intuitive, but generally displays a multiplicity of Nash equilibria. As a partial remedy, we define pairwise-Nash equilibrium, a variation of Nash equilibrium where players are allowed to deviate by pairs. Finally, we recall the definitions of perfect equilibrium (Selten 1975) and proper equilibrium (Myerson 1978), two Nash equilibrium refinements for normal form games.

Pairwise-stability Pairwise stable networks are robust to one-link deviations, where link severance is unilateral, while link creation is bilateral and under mutual consent of the two involved players.

Definition 3 A network $g \in \mathcal{G}$ is pairwise stable with respect to the network payoff function $u$ if and only if for all $i, j \in N$, if $i j \in g$ then both $m u_{i}(g, i j) \geq 0$ and $m u_{j}(g, i j) \geq 0$, while if $i j \notin g$ then $m u_{i}(g+i j, i j)>0$ implies $m u_{j}(g+i j, i j)<0$.

We denote by $P S(u)$ the set of pairwise stable networks with respect to $u$.

We comment on the relationship between pairwise-stability and $\alpha$-submodularity in own current links.

Suppose, first, that $u$ is $\alpha$-submodular on $P S(u)$. For any $g \in P S(u)$, by pairwisestability, the right-hand side of (1) is non-negative for all $i \in N$ and all $\ell \subseteq L_{i}(g)$. Then for all $i \in N$ and all $\ell \subseteq L_{i}(g), m u_{i}(g, \ell) \geq 0$. In words, when network payoffs are $\alpha$-submodular on $P S(u)$, no player would be better off by cutting any subset of her existing links simultaneously in a pairwise stable network.

Conversely, suppose that for all $g \in P S(u)$, all $i \in N$ and all $\ell \subseteq L_{i}(g)$, $m u_{i}(g, \ell) \geq 0$. Then, $u$ is 0 -submodular on $P S(u)$.

On pairwise stable networks, $\alpha$-submodularity in payoffs is thus equivalent to checking that no player wants to cut multiple links.

A normal form game of network formation This game is due to Myerson (1991). ${ }^{12}$ The set of players is $N$. All players $i \in N$ simultaneously announce the direct links they wish to form.

Formally, $S_{i}=\{0,1\}^{n-1}$ is $i$ 's set of pure strategies. Let $s_{i}=\left(s_{i 1}, \ldots s_{i, i-1}\right.$, $\left.s_{i, i+1}, \ldots s_{i n}\right) \in S_{i}$. Then, $s_{i j}=1$ if and only if $i$ chooses a direct link with $j \neq i$

\footnotetext{
11 See also Jackson and Watts (2002) for a dynamic foundation of pairwise-stability.

12 To quote Myerson: "Now consider a link-formation process in which each player independently writes down a list of players with whom he wants to form a link (...) and the payoff allocation is (...) for the graph that contains a link for every pair of players who have named each other." (p. 448)
} 
(and thus $s_{i j}=0$, otherwise). We assume that mutual consent is needed to establish a direct link, that is, $i j$ is created if and only if $s_{i j} s_{j i}=1$.

Let $S=S_{1} \times \cdots \times S_{n}$. A pure strategy profile $s=\left(s_{1}, \ldots, s_{n}\right) \in S$ induces an undirected network $g(s)$.

Pairwise-Nash equilibrium A pure strategy profile $s^{*}=\left(s_{1}^{*}, \ldots, s_{n}^{*}\right)$ is a Nash equilibrium of the game of network formation if and only if $u_{i}\left(g\left(s^{*}\right)\right) \geq u_{i}\left(g\left(s_{i}, s_{-i}^{*}\right)\right)$ for all $i \in N$ and all $s_{i} \in S_{i}$. The predictive power of Nash equilibrium is weak for the game of network formation. For instance, the empty network is always a Nash equilibrium. ${ }^{13}$ Building upon pairwise-stability, we further require that any mutually beneficial link be formed at equilibrium. Pairwise-Nash equilibrium networks are robust to bilateral commonly agreed one-link creation, and to unilateral multi-link severance.

Definition 4 A network $g \in \mathcal{G}$ is a pairwise-Nash equilibrium network with respect to the network payoff function $u$ if and only if there exists a pure Nash equilibrium strategy profile $s^{*}$ that supports $g$, that is, $g=g\left(s^{*}\right)$, and, for all $i, j \in N$, if $i j \notin g$, then $m u_{i}(g+i j, i j)>0$ implies $m u_{j}(g+i j, i j)<0$.

In other words, $g$ is a pairwise-Nash equilibrium network if it is both pairwise stable and a pure strategy Nash equilibrium outcome. We denote by $P N E(u)$ the set of pairwise-Nash equilibrium networks with respect to $u$.

Mixed Strategies Let $\Sigma_{i}=\Delta\left(\{0,1\}^{n-1}\right)$ be $i$ 's set of mixed strategies for the Myerson game of network formation, where $\Delta\left(\{0,1\}^{n-1}\right)$ denotes the set of probability distributions over $\{0,1\}^{n-1}$. A product of $n-1$ independent Bernoulli distributions is an example of a mixed strategy. More generally, a mixed strategy $\sigma_{i} \in \Sigma_{i}$ is a joint (multivariate Bernoulli) distribution that allows for rich correlation patterns in individual link announcements. In particular, given a mixed strategy $\sigma_{i}, i$ announces a link with $j$ with the following marginal probability: ${ }^{14}$

$$
\mu_{i j}^{\sigma_{i}}=\operatorname{Pr}^{\sigma_{i}}\left\{s_{i j}=1\right\}=\sum_{\left\{s \in\{0,1\}^{n-1} ; s_{i j}=1\right\}} \sigma_{i}(s) .
$$

Let $\Sigma=\Sigma_{1} \times \cdots \times \Sigma_{n}$. A mixed strategy $\sigma=\left(\sigma_{1}, \ldots, \sigma_{n}\right) \in \Sigma$ generates a probability distribution over $\mathcal{G}$, a random graph. ${ }^{15}$ Given $\sigma \in \Sigma$ and $g \in \mathcal{G}$, let $p_{\sigma}(g)$ be the probability that the network $g$ is formed when the mixed strategy profile $\sigma$ is

\footnotetext{
13 When nobody announces any link.

14 Note that, for $n \geq 3$, the collection of such marginal probabilities does not define univocally a mixed strategy. Fréchet (1951) gives an early and thorough account of the relationship between joint distributions (here, mixed strategies on the collection of all link announcements) and marginals (here, single link probabilities) when $n=3$.
}

15 See Jackson and Rogers (2006) for a relation between random graphs and individual incentives. 
played. Then the expected utility of player $i$ is:

$$
E u_{i}(\sigma)=\sum_{g \in \mathcal{G}} u_{i}(g) \cdot p_{\sigma}(g) .
$$

Perfect equilibrium We now define perfect equilibrium, a Nash equilibrium refinement. Let $\sigma \in \Sigma$ be a mixed strategy profile. We say the $\sigma$ is completely mixed if and only if for all $i \in N$ and all $s_{i} \in S_{i}$, we have $\sigma_{i}\left(s_{i}\right)>0$.

Definition 5 Let $\varepsilon>0$. A strategy profile $\sigma^{\varepsilon}$ is an $\varepsilon$-perfect equilibrium if it is a completely mixed strategy profile and, for all $i \in N$ and all $s_{i}, s_{i}^{\prime} \in S_{i}$, if $E u_{i}\left(s_{i}, \sigma_{-i}^{\varepsilon}\right)>$ $E u_{i}\left(s_{i}^{\prime}, \sigma_{-i}^{\varepsilon}\right)$, then $\sigma_{i}^{\varepsilon}\left(s_{i}^{\prime}\right)<\varepsilon$.

A strategy profile $\sigma \in \Sigma$ is a perfect equilibrium if there exists a sequence of strictly positive trembles $\left\{\varepsilon_{t}\right\}_{t \in \mathbb{N}}$ with limit 0 , and a sequence of $\varepsilon_{t}$-perfect equilibrium strategy profiles $\left\{\sigma^{\varepsilon_{t}}\right\}_{t \in \mathbb{N}}$ with limit $\sigma$.

In a perfect equilibrium, players best respond to their opponents' $\varepsilon$-deviations from the equilibrium strategies.

\section{Proper equilibrium}

Definition 6 A strategy profile $\sigma \in \Sigma$ is a proper equilibrium if there exists a sequence of strictly positive trembles $\left\{\varepsilon_{t}\right\}_{t \in \mathbb{N}}$ with limit 0 , and a sequence of $\varepsilon_{t}$-perfect equilibrium strategy profiles $\left\{\sigma^{\varepsilon_{t}}\right\}_{t \in \mathbb{N}}$ with limit $\sigma$, such that, for all $i \in N$, all $s_{i}, s_{i}^{\prime} \in S_{i}$, and all $t \in \mathbb{N}$ :

$$
E u_{i}\left(s_{i}, \sigma_{-i}^{\varepsilon_{t}}\right)>E u_{i}\left(s_{i}^{\prime}, \sigma_{-i}^{\varepsilon_{t}}\right) \text { implies that } \sigma_{i}^{\varepsilon_{t}}\left(s_{i}^{\prime}\right) \leq \varepsilon_{t} \cdot \sigma_{i}^{\varepsilon_{t}}\left(s_{i}\right)
$$

This refinement is due to Myerson (1978). In a proper equilibrium, players best respond to perturbations of their opponents' strategies, where costly mistakes are made with smaller probability. This hierarchy of mistakes differentiates properness from perfection. Myerson (1978) shows that every finite game in normal form has a proper equilibrium. We denote by $P R E(u)$ the set of proper equilibrium networks in pure strategies with respect to $u .^{16}$

\section{The results}

We first provide necessary and sufficient conditions on the payoff function $u$ for the set of pairwise stable networks and the set of pairwise-Nash equilibrium networks to coincide. We latter identify conditions under which the set of pairwise-Nash equilibrium networks and proper equilibrium networks coincide.

\footnotetext{
${ }^{16}$ We want to establish necessary and sufficient conditions such that the set of pairwise stable, pairwiseNash and proper equilibrium networks coincide. Given that both pairwise-stability and pairwise-Nash networks are only defined for pure strategies (that is, deterministic networks), we restrict attention to proper equilibria in pure strategies, but then, existence is not warranted. See Jackson and Watts (2001) for a perspective on the existence of pairwise stable networks.
} 


\section{Pairwise stable and pairwise-Nash equilibrium networks}

Theorem $1 P S(u)=P N E(u)$ if and only if $u$ is $\alpha$-submodular on PS $(u)$, for some $\alpha \geq 0$.

The proof derives from the simple observation that, when payoffs are $\alpha$-submodular on $P S(u)$ for some $\alpha \geq 0$, if a player does not benefit from severing any single link, then he does not benefit from cutting any group of links simultaneously. Robustness to unilateral and robustness to multilateral link severance are equivalent when marginal payoffs from existing links satisfy (1) on some particular set.

Note that $\alpha$-submodularity for some $\alpha \geq 0$ on $P S(u)$, implies $\alpha^{\prime}$-submodularity for any $\alpha>\alpha^{\prime}>0$. We can simply write,

Corollary $1 P S(u)=P N E(u)$ if and only if $u$ is 0-submodular on PS $(u)$.

Although Theorem 1 and Corollary 1 both characterize the equivalence between $P S(u)$ and $P N E(u)$, it is often easier in practice to establish $\alpha$-submodularity of a given network payoff for some particular $\alpha>0$ than 0 -submodularity. For instance, in Sect. 5 we consider the connections model (Jackson and Wolinsky 1996) and a model of information transmission (Calvó-Armengol 2004). In both cases gross network benefits and link costs are additively separable, and marginal link costs are constant. Then 1-submodularity of network payoffs is equivalent to 1-submodularity of gross network benefits, which we establish in Claims 1 (connections) and 3 (information transmission).

Pairwise-Nash and proper equilibrium networks We now define link-responsive network payoffs functions. We first start with a weak notion of link-responsiveness.

Definition 7 (weak link-responsiveness) The network payoff function $u$ is weakly link-responsive on $g$ if and only if for all $i \in N$ and all $\ell \subseteq L_{i}(g)$ such that $\ell \neq \varnothing$, we have $m u_{i}(g, \ell) \neq 0$.

Weak link-responsiveness states that no link removal is innocuous for the players directly involved. Network payoff functions that depend on some exogenous set of parameters (e.g., a constant marginal-link cost) are generically weakly linkresponsive. $^{17}$

We now strengthen this notion.

Definition 8 (strong link-responsiveness) The network payoff function $u$ is strongly link-responsive on $g$ if and only if for all $i \in N$, all $\ell \subseteq L_{i}(g)$ and all $\ell^{\prime} \subseteq L_{i}\left(g^{N} \backslash g\right)$ such that $g+\ell^{\prime}-\ell \neq g$, we have $u_{i}\left(g+\ell^{\prime}-\ell\right)-u_{i}(g) \neq 0$.

The strong version of link-responsiveness requires that no player is indifferent to a change in his set of direct links, whether due to formation, link removal, or a combination of both.

We finally define the set of links absent from a given network $g$ that generate a conflict of interest for the involved parties.

\footnotetext{
17 This is the case, for instance, for the model of information transmission.
} 
Definition 9 For all network payoff functions $u$, network $g \in \mathcal{G}$, and player $i \in N$, let:

$$
v_{i}(g, u)=\left\{i j \notin g: m u_{i}(g+i j, i j)<0 \text { and } m u_{j}(g+i j, i j)>0\right\} .
$$

This is the set of $i$ 's direct links not in $g$ with negative marginal returns to $i$ but positive marginal returns to the candidate partner.

We are now ready to state the equivalence between pairwise-Nash and proper equilibrium networks.

\section{Theorem 2 Let u be a network payoff function.}

(a) Let $g \in P N E(u)$. If $u$ is weakly link-responsive on $g$ and $\beta$-strongly supermodular for some $\beta>0$ in own new links on $v_{i}(g, u)$, for all $i \in N$, then $g \in \operatorname{PRE}(u)$.

(b) Let $g \in P R E(u)$. If $u$ is strongly link-responsive on $g$, then $g \in P N E(u)$.

The proof of the theorem is given in the next section. It consists of two propositions. The first one shows that strong link-responsiveness is enough for any proper equilibrium network to be also a pairwise-Nash equilibrium network. The proof is by contradiction. Consider a proper equilibrium network that is not pairwise stable, and a proper equilibrium strategy that supports it. ${ }^{18}$ Then, in this network, we can find a mutually beneficial link that is not formed, and that is not announced by either of the involved parties. We modify the proper equilibrium strategy by imposing that these two involved players announce this mutually beneficial link. These are the only link announcements that are modified. Consider a sequence of $\varepsilon$-proper equilibrium strategy profiles that converge to the proper equilibrium strategy. Using the order of magnitude of mistakes ranking in (8), we show that in this sequence of strategy profiles the modified strategy asymptotically payoff dominates the initial one, a violation of properness.

Strong link responsiveness is sufficient for a proper equilibrium network to be pairwise-stable, but moreover it is necessary for the general result. In the appendix we provide an example where strong link responsiveness is violated and a proper equilibrium network fails to be pairwise-stable.

Imposing weak link-responsiveness, the second proposition shows that $\beta$-strong supermodularity on $v_{i}(g, u)$ is sufficient to sustain pairwise-Nash equilibrium networks as a proper equilibrium outcome. Given $g \in P N E(u)$, we establish the result through a fixed-point argument on a suitably restricted strategy space.

$\beta$-strong supermodularity and weak link responsiveness are sufficient for a pairwiseNash network to be supported by a proper equilibrium. Moreover, $\beta$-strong supermodularity is necessary for the general result. In the appendix we provide an example where $\beta$-strong supermodularity is violated and a pairwise-Nash network can not be supported by a proper equilibrium.

Suppose now that every missing link from some network $g$ yields negative marginal returns to both parties, that is, both $m u_{i}(g+i j)<0$ and $m u_{j}(g+i j, i j)$, for all

\footnotetext{
18 Note that a proper equilibrium is a Nash equilibrium.
} 
$i j \notin g$. For this network, $v_{i}(g, u)=\varnothing$, for all $i \in N$. The $\beta$-strong supermodularity requirement is then equivalent to requiring that no link cutting is beneficial, that is, for all $i \in N$ and all $\ell \in L_{i}(g), m u_{i}(g, \ell)<0$, a condition that is trivially fulfilled at any Nash equilibrium. We can thus conclude the following.

Corollary 2 Consider some network payoff function u weakly link-responsive on some $g \in \mathcal{G}$ such that both $m u_{i}(g+i j, i j)<0$ and $m u_{j}(g+i j, i j)<0$, for all $i j \notin g$. Then, $g \in P N E(u)$ implies that $g \in P R E(u)$.

In the complete network, there is no room for extra links. Thus, a weakly linkresponsive network payoff function is also strongly link-responsive. Besides, $\beta$-strong supermodularity is trivially satisfied in this case. Altogether, we obtain the following.

Corollary 3 Suppose network payoff function u is weakly link-responsive on the complete network $g^{N}$. Then $g^{N}$ is a pairwise-Nash equilibrium network if and only if it is a proper equilibrium network.

\section{Examples}

In what follows, we first show with an example that perfect equilibrium outcomes need not be pairwise-Nash, even when the conditions in Theorem 2 hold. This justifies the need to resort to proper equilibrium, a refinement that differs from perfection by imposing a hierarchy on trembles related to the relative cost values associated with these mistakes.

Example (perfect equilibrium networks need not be pairwise-Nash) Let $N=\{1,2,3\}$ and consider the network payoffs defined below:
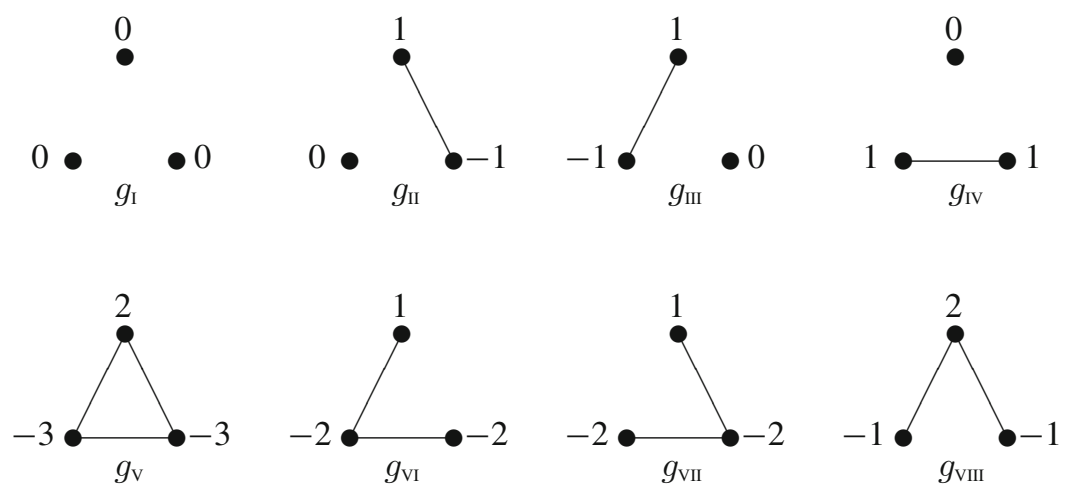

Denote by 1 the player at the top, and by 2 and 3 the players at the bottom, and let $S_{1}=\left\{\left(s_{12}, s_{13}\right) \in\{0,1\}^{2}\right\}, S_{2}=\left\{\left(s_{21}, s_{23}\right) \in\{0,1\}^{2}\right\}$ and $S_{3}=\left\{\left(s_{31}, s_{32}\right) \in\right.$ $\left.\{0,1\}^{2}\right\}$. 
The only two Nash equilibrium networks are $g_{\mathrm{I}}$ and $g_{\mathrm{IV}} \cdot{ }^{19}$ Clearly, the network payoff function $u$ is strongly link-responsive: any unilateral link addition to $g_{\mathrm{I}}$ and any unilateral link addition/removal to $g_{\mathrm{IV}}$ for any player in isolation has a strict impact on payoffs. So, by Theorem 2, any proper equilibrium network is also a pairwise-Nash equilibrium network. This inclusion is tight in the following sense: $g_{\mathrm{I}}$ is a perfect equilibrium network, whereas $g_{\mathrm{IV}}$ is the only pairwise-Nash equilibrium network. The inclusion in Theorem 2(a) thus applies to proper equilibrium networks, but not to perfect equilibrium networks.

More precisely, $g_{\mathrm{I}}$ is a perfect equilibrium network for the pure strategy $s^{*}=$ $((1,1),(0,0),(0,0))$ in which player 1 announces all the links, and players 2 and 3 do not announce any link. This strategy $s^{*}$ is the limit of the sequence of best-replying trembles of the perturbed game, where:

$$
\begin{aligned}
& \sigma_{1}^{\varepsilon}((0,0))=\sigma_{1}^{\varepsilon}((1,0))=\sigma_{1}^{\varepsilon}((0,1))=\varepsilon, \sigma_{1}^{\varepsilon}((1,1))=1-3 \varepsilon \\
& \sigma_{2}^{\varepsilon}((1,0))=\sigma_{2}^{\varepsilon}((0,1))=\sigma_{2}^{\varepsilon}((1,1))=\varepsilon, \sigma_{2}^{\varepsilon}((0,0))=1-3 \varepsilon \\
& \sigma_{3}^{\varepsilon}((1,0))=\sigma_{3}^{\varepsilon}((0,1))=\sigma_{3}^{\varepsilon}((1,1))=\varepsilon, \sigma_{3}^{\varepsilon}((0,0))=1-3 \varepsilon
\end{aligned}
$$

The reasons why (4) is a sequence of best-replying trembles of the perturbed game is the following.

First, for all $\left(s_{1}, s_{-1}\right) \in S, u_{1}\left(g\left((1,1), s_{-1}\right)\right) \geq u_{1}\left(g\left(s_{1}, s_{-1}\right)\right)$, the inequality being strict for some $\left(s_{i}, s_{-1}\right){ }^{20}$

Second, for all $s_{-2} \in S_{-2}$, we have $u_{2}\left(g\left((0,0), s_{-2}\right)\right) \geq u_{2}\left(g\left((1,0), s_{-2}\right)\right),{ }^{21}$ and $u_{2}\left(g\left((0,1), s_{-2}\right)\right) \geq u_{2}\left(g\left((1,1), s_{-2}\right)\right),{ }^{22}$ with a strict inequality for some $s_{-2} \in S_{-2}$. Similarly for player 3 . We are thus left to show that there exists some $\bar{\varepsilon}>0$ such that $E u_{2}\left((0,0), \sigma_{-2}^{\varepsilon}\right)>E u_{2}\left((0,1), \sigma_{-2}^{\varepsilon}\right)$ for all $0<\varepsilon<\bar{\varepsilon}$.

Note that, when link announcements are $\left((0,0), \sigma_{-2}^{\varepsilon}\right)$, the only possible networks that can be formed are $g_{\mathrm{I}}$ and $g_{\mathrm{II}}$. Given that player 2 gets a payoff equal to zero in both cases, $E u_{2}\left((0,0), \sigma_{-2}^{\varepsilon}\right)=0$. Instead, when link announcements are $\left((0,1), \sigma_{-2}^{\varepsilon}\right)$, four different networks may form, namely, $g_{\mathrm{I}}, g_{\mathrm{II}}, g_{\mathrm{IV}}$ and $g_{\mathrm{VII}}$. The network $g_{\mathrm{IV}}$ is formed with probability $\varepsilon+2 \varepsilon^{2}$, while the network $g_{\mathrm{VII}}$ arises with probability $\varepsilon-2 \varepsilon^{2}$. Therefore, $E u_{2}\left((0,1), \sigma_{-2}^{\varepsilon}\right)=-\varepsilon+6 \varepsilon^{2}<0$ for all $\varepsilon<1 / 6$.

Next, we apply our results to the connections model and the coauthor model, both due to Jackson and Wolinsky (1996), and to a model of information transmission due to Calvó-Armengol (2004).

\footnotetext{
${ }^{19} g_{\mathrm{I}}$ is a Nash equilibrium network for the following Nash equilibrium pure strategy profiles $s=$ $\left(s_{1}, s_{2}, s_{3}\right):((0,0),(0,0),(0,0)),((1,1),(0,0),(0,0)),((1,0),(0,0),(0,0)),((0,1),(0,0),(0,0))$. $g_{\mathrm{IV}}$ is a Nash equilibrium network for the following Nash equilibrium strategy profile: $((0,0),(0,1),(0,1))$. ${ }^{20}$ Indeed, notice that $u_{1}\left(g_{\mathrm{VIII}}\right)>\max \left\{u_{1}\left(g_{\mathrm{I}}\right), u_{1}\left(g_{\mathrm{II}}\right), u_{1}\left(g_{\mathrm{III}}\right)\right\}$, and $u_{1}\left(g_{\mathrm{V}}\right)>\max \left\{u_{1}\left(g_{\mathrm{IV}}\right)\right.$, $\left.u_{1}\left(g_{\mathrm{VI}}\right), u_{1}\left(g_{\mathrm{VII}}\right)\right\}$.

${ }^{21}$ Indeed, notice that $u_{2}\left(g_{\mathrm{I}}\right)>u_{2}\left(g_{\mathrm{III}}\right), u_{2}\left(g_{\mathrm{III}}\right)>u_{2}\left(g_{\mathrm{VIII}}\right), u_{2}\left(g_{\mathrm{IV}}\right)>u_{2}\left(g_{\mathrm{VI}}\right)$ and $u_{2}\left(g_{\mathrm{VII}}\right)>u_{2}\left(g_{\mathrm{V}}\right)$.

22 Indeed, notice that $u_{2}\left(g_{\mathrm{IV}}\right)>u_{2}\left(g_{\mathrm{VI}}\right)$ and $u_{2}\left(g_{\mathrm{VII}}\right)>u_{2}\left(g_{\mathrm{V}}\right)$.
} 
Example (connections model) This model is due to Jackson and Wolinsky (1996). Given some $\delta, c \in(0,1)$, define:

$$
u_{i}(g)=\sum_{j \in N} \delta^{d_{i j}(g)}-c n_{i}(g),
$$

where $n_{i}(g)=\left|L_{i}(g)\right|$ and $d_{i j}(g)$ is the geodesic distance between $i$ and $j$ on $g .{ }^{23} \mathrm{We}$ have the following result.

Claim 1 The connections model is 1 -submodular in own current links on $\mathcal{G}$.

Invoking Theorem 1, we can thus conclude that the set of pairwise stable and pairwise-Nash equilibrium networks coincide for the connections model.

Next, note that the connections model is generically weakly link-responsive. This is because the set of cost values $c$ for which some of the link marginal payoffs for some player are zero is finite, and thus of Lebesgue measure zero on $(0,1)$.

Jackson and Wolinsky (1996) characterizes the cost ranges for which the star network and the complete network are pairwise stable. Consider first the star network. The star encompassing all players is pairwise stable if and only if $\delta-\delta^{2}<c<\delta$. Besides, on this cost range, the peripheral players in the star get a negative return from any direct link with one another. Applying Corollary 2, we can thus conclude that the stars encompassing $n \geq 4$ players are proper equilibrium networks when $\delta-\delta^{2}<c<\delta$. Consider now the complete network. It is pairwise stable if and only if $c<\delta-\delta^{2}$. Invoking Corollary 3 we conclude that the complete network is a proper equilibrium network on $c<\delta-\delta^{2}$.

Example (coauthor model) This model is due to Jackson and Wolinsky (1996). Network payoffs are given by:

$$
u_{i}(g)=\left(1+\frac{1}{n_{i}(g)}\right) \sum_{i j \in g} \frac{1}{n_{j}(g)},
$$

if $\left|L_{i}(g)\right| \geq 1$, and $u_{i}(g)=0$, otherwise.

Claim 2 The coauthor model is 1-submodular in own current links on PS $(u)$.

By Theorem 1, the set of pairwise stable and pairwise-Nash equilibrium networks thus coincide. By Corollary 3, the complete network, which is pairwise stable (and pairwise-Nash), is also proper.

Instead, Theorem 2 cannot be used. Pairwise stable networks in the coauthor model consist of a sequence of fully connected networks such that, if we order this sequence by components' sizes, the square of the size of any component is smaller than the next size in this sequence. ${ }^{24}$ But coauthor payoffs decrease with the connectivity of one's contacts. Then, any player in a component gains by cutting any current links within his

\footnotetext{
23 That is, the length of the shortest path in $g$ between $i$ and $j$.

24 See Proposition 4 in Jackson and Wolinsky (1996).
} 
component, and rewiring with any player in a component of smaller size. Such links, though, are not formed because it is not in the interest of the players in the components of smaller size to consent to this rewiring process, implying that condition (2) does not hold.

Example (information transmission) This model is due to Calvó-Armengol (2004). Network payoffs are given by:

$$
u_{i}(g)=1-\prod_{i j \in g} p_{j i}-c n_{i}(g)
$$

where $p_{j i} \in(0,1)$, for all $i, j \in N$.

Claim 3 The information transmission model is 1-submodular in own current links on $\mathcal{G}$

By Theorem 1, the set of pairwise stable and pairwise-Nash equilibrium networks thus coincide.

\section{Discussion}

The Myerson game of network formation is a very simple and intuitive normal form game. It has been fruitfully used to analyze the formation of directed networks (Bala and Goyal 2000). But a number of major coordination problems arise when dealing with undirected networks. In the preceding literature, the formation of undirected networks has been studied using cooperative concepts such as pairwise-stability or pairwise-Nash equilibrium (Jackson 2004, Bloch and Jackson 2007).

We single out simple conditions on network payoffs such that pairwise stable networks do, in fact, coincide with a Nash equilibrium refinement for the Myerson game, the proper equilibrium. Our results can thus be read as providing a non-cooperative grounding to pairwise stability. Conversely, given the relative simplicity to find out pairwise stable networks in some cases, our results also provide a means to single out proper equilibrium networks of a normal form game with a multidimensional strategy space.

Our analysis also connects the computational issues in networks and game theory. Computing an agent's best-response profile is NP-hard for Nash equilibrium in the Myerson game, but polynomial-time solvable for pairwise stability. The same tradeoff exists for verifying that a network is an equilibrium. ${ }^{25}$

Theorem 2 shows that looking for pure strategy proper equilibrium in the Myerson game of network formation is equivalent to finding a pairwise-Nash network that has $\beta$-strong supermodular payoffs. We do not provide a result on the computational complexity of checking for $\beta$-strong supermodularity, but we show that it gives us proper equilibrium networks. Yamamoto (1993) provides a finite-time algorithm to find a proper equilibrium of a finite game. A relevant question is whether our results

\footnotetext{
25 We thank a referee for pointing this out.
} 
can help improve his algorithm so as to find a proper equilibrium of the Myerson game in polynomial time.

To some extent, our analysis also sheds light on the extensive form approach to network formation (and corresponding extensive form equilibrium refinements). Indeed, van Damme (1984) and Mailath et al. (1997) establish the equivalence between proper equilibria of a normal form game and the quasi-perfect equilibria of any extensive form equivalent of this normal form. This equivalence result, together with Theorems 1 and 2 , relates pairwise stable networks to the quasi-perfect equilibria of an extensive form game where players decide their link announcements sequentially.

\section{Main proofs}

Proof of Theorem 1 First, it is clear that $P N E(u) \subseteq P S(u)$. Hence, if $P S(u)=\emptyset$, the result follows. Suppose now that $P S(u) \neq \emptyset$, and let $g^{*} \in P S(u)$. Define:

$$
\phi\left(g^{*}, u\right) \in \min \left\{m u_{i}\left(g^{*}, i j\right): i j \in g, i \in N\right\} .
$$

Pairwise-stability implies that $\phi\left(g^{*}, u\right) \geq 0$. Suppose that for some $\alpha \geq 0, u$ is $\alpha$-submodular in own current links on $P S(u)$. Then, (1) implies that for all $i \in N$ and all $\ell \subseteq L_{i}\left(g^{*}\right), m u_{i}\left(g^{*}, \ell\right) \geq \alpha|\ell| \phi\left(g^{*}, u\right) \geq 0$, where $|\ell|$ is the cardinality of $\ell$. Moreover, by definition of pairwise-stability, for all $i, j \in N$ such that $i j \notin L_{i}\left(g^{*}\right)$, if $m u_{i}\left(g^{*}+i j, i j\right)>0$, then $m u_{j}\left(g^{*}+i j, i j\right)<0$. Therefore, $g^{*} \in P N E(u)$.

Now, suppose that there exists $g^{*} \in P S(u)$ such that, for all $\alpha \geq 0$, (1) does not hold for $g^{*}$. Then, there exist $i \in N$ and $\ell \subseteq L_{i}\left(g^{*}\right)$ such that $m u_{i}\left(g^{*}, \ell\right)<0$, implying that $g^{*} \notin P N E(u)$.

Proof of Theorem 2 We decompose the proof in two parts. Proposition 2 establishes that $g \in P R E(u)$ implies $g \in P N E(u)$ when $u$ is strongly link-responsive on $g$. Proposition 3 then shows that $g \in P N E(u)$ implies that $g \in \operatorname{PRE}(u)$ if $u$ is weakly link-responsive on $g$, and $\beta$-strongly supermodular in own new links on $v_{i}(g, u)$, for all $i \in N$, and for some $\beta>0$.

Proposition 2 Let $g \in P R E(u)$. If $u$ is strongly link-responsive on $g$, then $g \in$ PNE (u).

Proof Let $u$ be strongly link-responsive on $g^{*} \in P R E(u)$. We prove that in a pure strategy proper equilibrium $s^{*}$, if $m u_{i}\left(g^{*}+i j, i j\right)>0$ then $s_{i j}^{*}=1$, for all $i \in N$. This implies that in a proper equilibrium network no absent link is mutually beneficial. Hence, all proper equilibrium networks, which are Nash networks by definition, are also pairwise-Nash.

Let $g^{*} \in \operatorname{PRE}(u)$, and let $s^{*}$ be a pure strategy proper equilibrium that supports it. Then, $g^{*}=g\left(s^{*}\right)$. Let $\left\{\sigma^{\varepsilon_{t}}\right\}_{t \in \mathbb{N}}$ be a sequence of completely mixed strategy profiles such that $\lim _{t \rightarrow+\infty} \varepsilon_{t}=0, \lim _{t \rightarrow+\infty} \sigma^{\varepsilon_{t}}\left(s^{*}\right)=1$, and $\left\{\sigma^{\varepsilon_{t}}\right\}_{t \in \mathbb{N}}$ satisfies (8).

For a contradiction, suppose that there exists $i j \notin g$ such that both $m u_{i}\left(g^{*}+\right.$ $i j, i j)>0$ and $s_{i j}^{*}=0$. Given that $s^{*}$ is also a Nash equilibrium strategy and that $i j \notin g^{*}$, then $s_{j i}^{*}=0$, as otherwise $s_{i j}^{*}=0$ could not be part of a best-response strategy by player $i$. 
Let $s_{i}^{\prime}$ be the strategy of player $i$ that differs from $s_{i}^{*}$ only in its $j$ th coordinate, meaning that $s_{i j}^{*}=0$ but $s_{i j}^{\prime}=1$, while $s_{i k}^{\prime}=s_{i k}^{*}$, for all $k \neq j$. In $s_{i}^{\prime}$, player $i$ announces exactly the same links as in $s_{i}^{*}$ plus an extra link with player $j$. This extra link is not reciprocated by player $j$ in $s_{j}^{*}$.

Define $s_{j}^{\prime}$ accordingly.

For all $t \in \mathbb{N}$, we have:

$$
E u_{i}\left(s_{i}^{\prime}, \sigma_{-i}^{\varepsilon_{t}}\right)-E u_{i}\left(s_{i}^{*}, \sigma_{-i}^{\varepsilon_{t}}\right)=\sum_{s_{-i} \in S_{-i}} \sigma_{-i}^{\varepsilon_{t}}\left(s_{-i}\right)\left[u_{i}\left(g\left(s_{i}^{\prime}, s_{-i}\right)\right)-u_{i}\left(g\left(s_{i}^{*}, s_{-i}\right)\right)\right] .
$$

For all $s_{-i}$ such that $s_{j i}=0, g\left(s_{i}^{\prime}, s_{-i}\right)=g\left(s_{i}^{*}, s_{-i}\right)$, and thus $u_{i}\left(g\left(s_{i}^{\prime}, s_{-i}\right)\right)=$ $u_{i}\left(g\left(s_{i}^{*}, s_{-i}\right)\right)$. Therefore,

$$
E u_{i}\left(s_{i}^{\prime}, \sigma_{-i}^{\varepsilon_{t}}\right)-E u_{i}\left(s_{i}^{*}, \sigma_{-i}^{\varepsilon_{t}}\right)=\sum_{s_{-i} \in S_{-i}: s_{i j}=1} \sigma_{-i}^{\varepsilon_{t}}\left(s_{-i}\right)\left[u_{i}\left(g\left(s_{i}^{\prime}, s_{-i}\right)\right)-u_{i}\left(g\left(s_{i}^{*}, s_{-i}\right)\right)\right] .
$$

Let $s_{-i} \in S_{-i}$ with $s_{j i}=1$. Note that $i j \notin g\left(s_{i}^{*}, s_{-i}\right)$ while $g\left(s_{i}^{\prime}, s_{-i}\right)=$ $g\left(s_{i}^{*}, s_{-i}\right)+i j$. Hence, the two networks $g\left(s_{i}^{*}, s_{-i}\right)$ and $g\left(s_{i}^{\prime}, s_{-i}\right)$ differ only in the link $i j$, which exists in the latter, but not in the former. Define

$$
\mathcal{G}\left(s_{i}^{*}\right)=\left\{g\left(s_{i}^{*}, s_{-i}\right): s_{-i} \in S_{-i}, s_{j i}=1\right\} .
$$

We can write:

$$
E u_{i}\left(s_{i}^{\prime}, \sigma_{-i}^{\varepsilon_{t}}\right)-E u_{i}\left(s_{i}^{*}, \sigma_{-i}^{\varepsilon_{t}}\right)=\sum_{g \in \mathcal{G}\left(s_{i}^{*}\right)} \tau_{\varepsilon_{t}}(g) m u_{i}(g+i j, i j)
$$

where

$$
\tau_{\varepsilon_{t}}(g)=\sum_{\substack{s_{-i} \in S_{-i}: s_{j i}=1 \\ g\left(s_{i}^{*}, s_{-i}\right)=g}} \sigma_{-i}^{\varepsilon_{t}}\left(s_{-i}\right), \text { for all } g \in \mathcal{G}\left(s_{i}^{*}\right)
$$

Lemma $1 \tau_{\varepsilon_{t}}(g) \in o\left(\tau_{\varepsilon_{t}}\left(g^{*}\right)\right)$, for all $g \in \mathcal{G}\left(s_{i}^{*}\right), g \neq g^{*}$.

Proof of Lemma 1 By definition, $\tau_{\varepsilon_{t}}(g) \in o\left(\tau_{\varepsilon_{t}}\left(g^{*}\right)\right)$ is equivalent to $\lim _{t \rightarrow+\infty} \tau_{\varepsilon_{t}}(g) /$ $\tau_{\varepsilon_{t}}\left(g^{*}\right)=0$, for all $g \in \mathcal{G}\left(s_{i}^{*}\right), g \neq g^{*}{ }^{26}$ This is implied by properness, as detailed below.

\footnotetext{
26 Note that $\lim _{t \rightarrow+\infty} \sigma_{-i}^{\varepsilon_{t}}\left(s_{-i}\right)=0$, for all $s_{-i} \in S_{-i}$ such that $s_{j i}=1$. Therefore, $\lim _{t \rightarrow+\infty} \tau_{\varepsilon_{t}}(g)=$ 0 , for all $g \in \mathcal{G}\left(s_{i}^{*}\right)$, including $g=g^{*}$. Establishing that $\tau_{\varepsilon_{t}}(g) \in o\left(\tau_{\varepsilon_{t}}\left(g^{*}\right)\right)$, for all $g \in \mathcal{G}\left(s_{i}^{*}\right), g \neq g^{*}$ is thus equivalent to showing that the rate of convergence to zero of $\tau_{\varepsilon_{t}}(g), g \neq g^{*}$ is at least one order of magnitude higher than that of $\tau_{\varepsilon_{t}}\left(g^{*}\right)$.
} 
For each $k \in N$, we partition the strategy set $S_{k}$ into two disjoint sets $S_{k}^{+}$and $S_{k}^{-}$ where:

$$
\left\{\begin{array}{l}
S_{k}^{+}=\left\{s_{k} \in S_{k}: u_{k}\left(g\left(s_{k}, s_{-k}^{*}\right)\right) \geq u_{k}\left(g^{*}\right)\right\} \\
S_{k}^{-}=\left\{s_{k} \in S_{k}: u_{k}\left(g\left(s_{k}, s_{-k}^{*}\right)\right)<u_{k}\left(g^{*}\right)\right\}
\end{array}\right. \text {. }
$$

It is plain that $S_{k}=S_{k}^{+} \cup S_{k}^{-}$and that $S_{k}^{+} \cap S_{k}^{-}=\varnothing$. Given that $s^{*}$ is a Nash equilibrium strategy supporting $g^{*}$, we have $u_{k}\left(g\left(s_{k}, s_{-k}^{*}\right)\right)=u_{k}\left(g^{*}\right)$, for all $s_{k} \in S_{k}^{+}$. Given that $u$ is strongly link-responsive on $g^{*}$, we then have $g\left(s_{k}, s_{-k}^{*}\right)=g^{*}$, for all $s_{k} \in S_{k}^{+}$.

Note that $s_{j}^{\prime} \in S_{j}^{+}$and that $s_{k}^{*} \in S_{k}^{+}$, for all $k \in N$.

Each player's expected payoff is continuous in the vector of other players' (completely) mixed strategies. Besides, $S_{k}$ is finite and $\lim _{t \rightarrow+\infty} \sigma^{\varepsilon_{t}}=s^{*}$. We thus conclude that there exists $t_{k}$ such that, for all $t \geq t_{k}$, all $s_{k}^{+} \in S_{k}^{+}$and all $s_{k}^{-} \in S_{k}^{-}$, we have $u_{k}\left(g\left(s_{k}^{+}, \sigma_{-k}^{\varepsilon_{t}}\right)\right)>u_{k}\left(g\left(s_{k}^{-}, \sigma_{-k}^{\varepsilon_{t}}\right)\right)$. Given that $\left\{\sigma^{\varepsilon_{t}}\right\}_{t \in \mathbb{N}}$ is a sequence of completely mixed strategy profiles that satisfy (8), we obtain the following result.

Claim 4 For all $k \in N$, there exists some integer $t_{k}$ such that, for all $t \geq t_{k}$, all $s_{k}^{+} \in S_{k}^{+}$and all $s_{k}^{-} \in S_{k}^{-}$we have $\sigma_{k}^{\varepsilon_{t}}\left(s_{k}^{-}\right) \leq \varepsilon_{t} . \sigma_{k}^{\varepsilon_{t}}\left(s_{k}^{+}\right)$.

Define now:

$$
\mathcal{G}^{\prime}=\left\{g \in \mathcal{G}\left(s_{i}^{*}\right): g \neq g^{*}, g=g\left(s_{j}, s_{-j}^{*}\right), s_{j} \in S_{j}, s_{j i}=1\right\} .
$$

In words, $\mathcal{G}^{\prime}$ is the set of networks derived from $g^{*}$ under $s^{*}$ where only player $j$ makes a mistake (including always the announcement of the link $i j$ ). Define also $\mathcal{G}^{\prime \prime}=\mathcal{G}^{\prime} \backslash \mathcal{G}\left(s_{i}^{*}\right) \cup\left\{g^{*}\right\}$. The set $\mathcal{G}^{\prime \prime}$ covers all the networks derived from $g^{*}$ under $s^{*}$ where at least some other player (besides $j$ ) makes a mistake.

Clearly, $\mathcal{G}\left(s_{i}^{*}\right)=\mathcal{G}^{\prime} \cup \mathcal{G}^{\prime \prime} \cup\left\{g^{*}\right\}$, and $\mathcal{G}^{\prime} \cap \mathcal{G}^{\prime \prime}=\varnothing$. Let $g \in \mathcal{G}^{\prime}$. Define $S_{-i}^{-1}(g)=$ $\left\{s_{-j} \in S_{-j}: s_{j i}=1, g\left(s_{i}^{*}, s_{-i}\right)=g\right\}$. This is the set of all pure strategy profiles that support $g$ and where $j$ announces the link $i j$. Then, $\tau_{\varepsilon_{t}}(g)=\sum_{s_{-i} \in S_{-i}^{-1}(g)} \sigma_{-i}^{\varepsilon_{t}}\left(s_{-i}\right)$.

Let $\left(s_{j}, s_{-i-j}^{*}\right) \in S_{-i}^{-1}(g)$. Such a strategy profile exists by definition of $\mathcal{G}^{\prime}$. We have $g=g\left(s_{i}^{*}, s_{j}, s_{-i-j}^{*}\right)=g\left(s_{j}, s_{-j}^{*}\right)$. By definition of $\mathcal{G}^{\prime}, g \neq g^{*}$. Strong linkresponsiveness then implies that $s_{j} \in S_{j}^{-}$. Recalling that $s_{j}^{\prime} \in S_{j}^{+}$, we conclude from Claim 4 that $\sigma_{j}^{\varepsilon_{t}}\left(s_{j}\right) \in o\left(\sigma_{j}^{\varepsilon_{t}}\left(s_{j}^{\prime}\right)\right)$. Noting that $\sigma_{-i}^{\varepsilon_{t}}\left(s_{-i}\right)=\sigma_{j}^{\varepsilon_{t}}\left(s_{j}\right) \sigma_{-i-j}^{\varepsilon_{t}}\left(s_{-i-j}\right)$ for all $s_{-i} \in S_{-i}$, we obtain $\sigma_{-i}^{\varepsilon_{t}}\left(s_{j}, s_{-i-j}^{*}\right) \in o\left(\sigma_{-i}^{\varepsilon_{t}}\left(s_{j}^{\prime}, s_{-i-j}^{*}\right)\right)$

More generally, let $\widetilde{s}_{-i}=\left(\widetilde{s}_{j}, \widetilde{s}_{-i-j}\right) \in S_{-i}^{-1}(g)$. We distinguish two cases.

First, suppose that $\widetilde{s}_{j} \in S_{j}^{-}$. Then, we deduce mutatis mutandis that $\sigma_{j}^{\varepsilon_{t}}\left(\widetilde{s}_{j}\right) \in$ $o\left(\sigma_{j}^{\varepsilon_{t}}\left(s_{j}^{\prime}\right)\right)$. Given that $\lim _{t \rightarrow \infty} \sigma_{-i-j}^{\varepsilon_{t}}\left(s_{-i-j}^{*}\right)=1 \geq \lim _{t \rightarrow \infty} \sigma_{-i-j}^{\varepsilon_{t}}\left(s_{-i-j}\right)$ for arbitrary $s_{-i-j}$, we can write that $\sigma_{-i-j}^{\varepsilon_{t}}\left(\widetilde{s}_{-i-j}\right) \in O\left(\sigma_{-i-j}^{\varepsilon_{t}}\left(s_{-i-j}^{*}\right)\right)$. Therefore, $\sigma_{-i}^{\varepsilon_{t}}\left(\widetilde{s}_{-i}\right) \in o\left(\sigma_{-i}^{\varepsilon_{t}}\left(s_{j}^{\prime}, s_{-i-j}^{*}\right)\right)$. 
Second, suppose that $\widetilde{s}_{j} \in S_{j}^{+}$. By strong link-responsiveness, this is equivalent to $g\left(\widetilde{s}_{j}, s_{-j}^{*}\right)=g^{*}$. Therefore, $j^{\prime}$ s link announcements in $\widetilde{s}_{-i}$ alone do not suffice to generate $g=g\left(s_{i}^{*}, \widetilde{s}_{-i}\right)$. Still, the network $g\left(s_{i}^{*}, \widetilde{s}_{-i}\right) \in \mathcal{G}^{\prime}$ differs from $g^{*} .{ }^{27}$ It thus is the case that $\widetilde{s}_{k} \neq s_{k}^{*}$ for some $k \in N, k \neq i, k \neq j$. But, by definition, all networks in $\mathcal{G}^{\prime}$ can be obtained from $g^{*}$ by $j$ 's mistakes alone. Therefore, there exists some $k$, $k \neq i, k \neq j$ whose link announcement $\widetilde{s}_{k} \neq s_{k}^{*}$ changes $g^{*}$ in a way that could also be achieved by $j$ 's mistakes alone. The only network changes due to unilateral mistakes are link severance. Therefore, there exists $k$ such that $j k \in g^{*}$ but $j k \notin g\left(\widetilde{s}_{k}, s_{-k}^{*}\right)$. This, in turn, implies that $\widetilde{s}_{k} \in S_{k}^{-}$. By Claim 4, $\sigma_{k}^{\varepsilon_{t}}\left(\widetilde{s}_{k}\right) \in o\left(\sigma_{k}^{\varepsilon_{t}}\left(s_{k}^{*}\right)\right)$. Again, noting that $\sigma_{-i-k}^{\varepsilon_{t}}\left(\widetilde{s}_{-i-k}\right) \in O\left(\sigma_{-i-k}^{\varepsilon_{t}}\left(s_{-i-k}^{*}\right)\right)$, we conclude that $\sigma_{-i}^{\varepsilon_{t}}\left(\widetilde{s}_{-i}\right) \in o\left(\sigma_{-i}^{\varepsilon_{t}}\left(s_{-i}^{*}\right)\right)$.

We have thus shown that for all $g \in \mathcal{G}^{\prime}$ and all $\widetilde{s}_{-i} \in S_{-i}^{-1}(g)$, either $\sigma_{-i}^{\varepsilon_{t}}\left(\widetilde{S}_{-i}\right) \in$ $o\left(\sigma_{-i}^{\varepsilon_{t}}\left(s_{j}^{\prime}, s_{-i-j}^{*}\right)\right)$ or $\sigma_{-i}^{\varepsilon_{t}}\left(\widetilde{s}_{-i}\right) \in o\left(\sigma_{-i}^{\varepsilon_{t}}\left(s_{-i}^{*}\right)\right)$. But both $\left(s_{j}^{\prime}, s_{-i-j}^{*}\right), s_{-i}^{*} \in S_{-i}^{-1}\left(g^{*}\right)$. Therefore, $\tau_{\varepsilon_{t}}(g) \in o\left(\tau_{\varepsilon_{t}}\left(g^{*}\right)\right)$, for all $g \in \mathcal{G}^{\prime}$.

Let $g \in \mathcal{G}^{\prime \prime}$.

Let $\widetilde{S}_{-i} \in S_{-i}^{-1}(g)$. Recall that in $\mathcal{G}^{\prime \prime}$ at least two players make a mistake. Therefore, there exist $\widehat{g} \in \mathcal{G}^{\prime}$ and $\widehat{s}_{-i} \in S_{-i}^{-1}(\widehat{g})$, such that $\sigma_{-i}^{\varepsilon_{t}}\left(\widetilde{s}_{-i}\right) \in O\left(\sigma_{-i}^{\varepsilon_{t}}\left(\widehat{s}_{-i}\right)\right)$, and the conclusion follows.

We are now ready to establish Proposition 2.

The mixed strategy profiles $\left\{\sigma^{\varepsilon_{t}}\right\}_{t \in \mathbb{N}}$ have full support, implying that $\tau_{\varepsilon_{t}}(g) \neq 0$, for all $g \in \mathcal{G}\left(s_{i}^{*}\right)$ and $t \in \mathbb{N}$. We then deduce from (6) that

$$
E u_{i}\left(s_{i}^{\prime}, \sigma_{-i}^{\varepsilon_{t}}\right)>E u_{i}\left(s_{i}^{*}, \sigma_{-i}^{\varepsilon_{t}}\right)
$$

is equivalent to

$$
m u_{i}\left(g^{*}+i j, i j\right)+\sum_{g \in \mathcal{G}\left(s_{i}^{*}\right), g \neq g^{*}} \frac{\tau_{\varepsilon_{t}}(g)}{\tau_{\varepsilon_{t}}\left(g^{*}\right)} m u_{i}(g+i j, i j)>0 .
$$

By assumption, $m u_{i}\left(g^{*}+i j, i j\right)>0$. By Lemma 1 , we then conclude that there exists some integer $T$ such that $E u_{i}\left(s_{i}^{\prime}, \sigma_{-i}^{\varepsilon_{t}}\right)>E u_{i}\left(s_{i}^{*}, \sigma_{-i}^{\varepsilon_{t}}\right)$, for all $t \geq T$. But then, given that $\sigma^{\varepsilon_{t}}$ is an $\varepsilon_{t}$-proper equilibrium, there exists some integer $T^{\prime}$ such that $\sigma_{i}^{\varepsilon_{t}}\left(s_{i}^{*}\right) \leq \varepsilon_{t} \sigma_{i}^{\varepsilon_{t}}\left(s_{i}^{\prime}\right)$, for all $t \geq T^{\prime}$, which in turn implies that $\lim _{t \rightarrow+\infty} \sigma_{i}^{\varepsilon_{t}}\left(s_{i}^{*}\right)=0$. This is a contradiction.

Proposition 3 Let $g \in P N E(u)$. If $u$ is weakly link-responsive on $g$ and $\beta$-strongly supermodular in own new links on $v_{i}(g, u)$, for all $i \in N$, then $g \in P R E(u)$.

Proof We first introduce some notation. Let $g \in \mathcal{G}$. Let $0<\epsilon<1$. For all $i \in N$, define $\widetilde{\Sigma}_{i}^{\epsilon}=\left\{\sigma_{i} \in \Sigma_{i}: \sigma_{i}\left(s_{i}\right) \geq \epsilon^{2^{n-1}} / 2^{n-1}\right.$, for all $\left.s_{i} \in S_{i}\right\}$.

Let now $0<\delta<1$. For all $i \in N$, let

$$
\Sigma_{i}^{\delta}(g)=\Sigma_{i}^{\delta, 1}(g) \cap \Sigma_{i}^{\delta, 2}(g) \cap \Sigma_{i}^{\delta, 3}(g),
$$

$\overline{27 \text { Recall that, by definition, } g^{*}} \notin \mathcal{G}^{\prime}$. 
where the sets $\Sigma_{i}^{\delta, k}(g), k=1,2,3$ are defined as follows.

First, $\Sigma_{i}^{\delta, 1}(g)=\left\{\sigma_{i} \in \Sigma_{i}\right.$ : if $s_{i} \in S_{i}$ is such that $s_{i j}=0$ for some $i j \in g$, then $\left.\sigma_{i}\left(s_{i}\right) \leq \delta\right\}$. Second, $\Sigma_{i}^{\delta, 2}(g)=\left\{\sigma_{i} \in \Sigma_{i}\right.$ : if $s_{i} \in S_{i}$ is such that $s_{i j}=1$ and $m u_{i}(g+i j, i j)<0$ for some $i j \notin g$, then $\left.\sigma_{i}\left(s_{i}\right) \leq \delta\right\}$. To define $\Sigma_{i}^{\delta, 3}(g)$ is more involved.

Let $s_{i} \in S_{i}$ such that $s_{i j}=1$ for some $i j \in v_{i}(g, u)$. We define the following pure strategy announcement $s_{i}^{0}\left(g, s_{i}, i j\right)$ for player $i$ :

$$
s_{i k}^{0}\left(g, s_{i}, i j\right)= \begin{cases}1, & \text { if } i k \in g \text { or } k=j \\ 0, & \text { if } i k \notin g, i k \notin v_{i}(g, u) \text { but } m u_{k}(g+i k, i k)<0 \\ s_{i k}, & \text { otherwise. }\end{cases}
$$

Among all the links mistakenly announced under $s_{i}$, only the link $i j$ is still announced under $s_{i}^{0}\left(g, s_{i}, i j\right)$.

Let then $\Sigma_{i}^{\delta, 3}(g)=\left\{\sigma_{i} \in \Sigma_{i}\right.$ : if $s_{i} \in S_{i}$ is such that $\exists i j \notin g, i j \in v_{i}(g, u)$ and $s_{i j}=1$, and $\exists k \neq j$ such that either $i k \in g$ and $s_{i k}=0$, or $i k \notin g, m u_{i}(g+i k, i k)<0$ and $s_{i k}=1$, or both, then $\left.\sigma_{i}\left(s_{i}\right) \leq \delta \sigma_{i}\left(s_{i}^{0}\left(g, s_{i}, i j\right)\right)\right\}$.

For player $i$, let $\widetilde{s}_{i}$ be so that

$$
\widetilde{s}_{i j}= \begin{cases}1, & \text { if } i j \in g \\ 0, & \text { if } i j \notin g .\end{cases}
$$

Then for all $i$ and all $k=1,2,3$, the pure strategy $\widetilde{s}_{i} \in \Sigma_{i}^{\delta, k}(g)$. Hence, for all $i$, $\Sigma_{i}^{\delta}(g) \neq \varnothing$.

Notice that the restrictions on the strategies in the definition of both $\widetilde{\Sigma}_{i}^{\epsilon}$ and $\Sigma_{i}^{\delta}(g)$ are weak linear inequalities, so that $\widetilde{\Sigma}_{i}^{\epsilon}, \Sigma_{i}^{\delta}$ and $\widetilde{\Sigma}_{i}^{\epsilon} \cap \Sigma_{i}^{\delta}$ are all compact and convex sets, for all $i \in N$.

Given some network $g$, and some $\sigma_{-i} \in \widetilde{\Sigma}_{-i}^{\epsilon} \cap \Sigma_{-i}^{\delta}(g)$, we now consider the following best-response correspondence:

$$
\begin{aligned}
& r_{i}^{g}\left(\sigma_{-i}\right)=\left\{\sigma_{i} \in \widetilde{\Sigma}_{i}^{\epsilon}: \sigma_{i}\left(s_{i}\right) \leq \epsilon \sigma_{i}\left(s_{i}^{\prime}\right), \text { for all } s_{i}, s_{i}^{\prime} \in S_{i}\right. \text { such that } \\
& \left.\quad E u_{i}\left(s_{i}, \sigma_{-i}\right)<E u_{i}\left(s_{i}^{\prime}, \sigma_{-i}\right)\right\}
\end{aligned}
$$

Clearly, $r_{i}^{g}(\cdot)$ is convex-valued, compact-valued and upper-hemicontinuous.

Given a $\sigma_{-i} \in \widetilde{\Sigma}_{-i}^{\epsilon} \cap \Sigma_{-i}^{\delta}(g)$, define $\rho_{i}^{g}\left(\sigma_{-i}\right)=r_{i}^{g}\left(\sigma_{-i}\right) \cap \Sigma_{i}^{\delta}(g)$. By definition, $\rho_{i}^{g}: \widetilde{\Sigma}_{i}^{\epsilon} \cap \Sigma_{i}^{\delta}(g) \rightarrow \widetilde{\Sigma}_{i}^{\epsilon} \cap \Sigma_{i}^{\delta}(g)$. Given that $\Sigma_{i}^{\delta}(g)$ is convex, compact, and given the properties of $r_{i}^{g}$ enunciated above, we deduce that $\rho_{i}^{g}$ is convex and compact-valued, and upper-hemicontinuous.

We now show that $\rho_{i}^{g}\left(\sigma_{-i}\right)$ is not empty-valued when $\sigma_{-i} \in \widetilde{\Sigma}_{-i}^{\epsilon} \cap \Sigma_{-i}^{\delta}(g)$.

Fix one such $\sigma_{-i}$. For each $s_{i} \in S_{i}$, let $\gamma^{\sigma_{-i}}\left(s_{i}\right) \geq 0$ be the number of strategies $s_{i}^{\prime} \in S_{i}$ such that $E u_{i}\left(s_{i}, \sigma_{-i}\right)<E u_{i}\left(s_{i}^{\prime}, \sigma_{-i}\right)$. By definition, $\gamma^{\sigma_{-i}}: S_{i} \rightarrow \mathbb{N}$ is a single-valued function, and $\gamma^{\sigma_{-i}}\left(s_{i}\right)=0$ if and only if $s_{i}$ is a best-response to $\sigma_{-i}$. 
Define now the following strategy $\widehat{\sigma}_{i}^{\sigma_{-i}}$ :

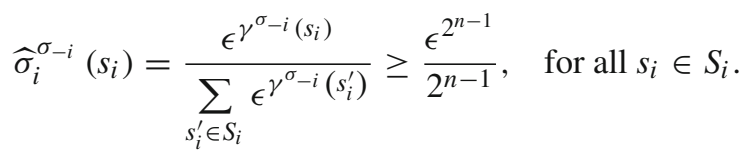

Therefore, by definition, $\widehat{\sigma}_{i}^{\sigma_{-i}} \in \widetilde{\Sigma}_{i}^{\epsilon}$ and $\widehat{\sigma}_{i}^{\sigma_{-i}} \in r_{i}^{g}\left(\sigma_{-i}\right)$. If we can show that $\widehat{\sigma}_{i}^{\sigma_{-i}} \in \widetilde{\Sigma}_{i}^{\epsilon} \cap \Sigma_{i}^{\delta}(g)$ for some $\delta>0$, then we will be able to conclude that $\widehat{\sigma}_{i}^{\sigma_{-i}} \in$ $\tilde{\Sigma}_{i}^{\epsilon} \cap \Sigma_{-i}^{\delta}(g)$, and thus $\widehat{\sigma}_{i}^{\sigma_{-i}} \in \rho_{i}^{g}\left(\sigma_{-i}\right)$.

To establish the existence of such a $\delta>0$, we need two intermediate results.

Lemma 2 Let $g^{*} \in P N E(u)$ and $\sigma_{-i} \in \widetilde{\Sigma}_{-i}^{\epsilon} \cap \Sigma_{-i}^{\delta}\left(g^{*}\right)$. Suppose that, for some $s_{i} \in S_{i}$, we have $E u_{i}\left(s_{i}^{\prime}, \sigma_{-i}\right)<E u_{i}\left(s_{i}, \sigma_{-i}\right)$, for all $s_{i}^{\prime} \in S_{i}$, Then, there exists a $\delta>0$ such that, for all $i j \in g^{*}$ we have $s_{i j}=1$, while for all $i j \notin g^{*}$ such that $m u_{i}\left(g^{*}+i j, i j\right)<0$, we have $s_{i j}=0$.

Proof of Lemma 2 Let $s_{i} \in S_{i}$ be such that there exists $i j \in g^{*}$ for which $s_{i j}=0$, or there exists $i j \notin g^{*}$ for which both $s_{i j}=1$ and $m u_{i}\left(g^{*}+i j, i j\right)<0 .{ }^{28}$ For a contradiction, suppose that there exists $\sigma_{-i} \in \widetilde{\Sigma}_{-i}^{\epsilon} \cap \Sigma_{-i}^{\delta}\left(g^{*}\right)$ such that, for all $\delta>0$, we have $E u_{i}\left(s_{i}^{\prime}, \sigma_{-i}\right) \leq E u_{i}\left(s_{i}, \sigma_{-i}\right)$, for all $s_{i}^{\prime} \in S_{i}$. We distinguish three cases.

Case 1: Player $i$ cuts a link. There exists $i j \in g^{*}$ for which $s_{i j}=0$. Let $\ell_{1}\left(s_{i}\right)=$ $\left\{i j \in g^{*}: s_{i j}=0\right\}$. Clearly, $\ell_{1}\left(s_{i}\right) \neq \varnothing$. Let $s_{i}^{\prime}$ be given by:

$$
s_{i j}^{\prime}= \begin{cases}1, & \text { if } i j \in g^{*}, \\ 0, & \text { if } i j \notin g^{*}\end{cases}
$$

Given that the strategy space is finite, there exists $\xi \in \mathbb{N}, \xi \neq 0$, such that $\operatorname{Pr}\left\{g\left(s_{i}, \sigma_{-i}\right)=g^{*}-\ell_{1}\left(s_{i}\right)\right\} \geq 1-\xi \delta$, for all $\sigma_{-i} \in \widetilde{\Sigma}_{-i}^{\epsilon} \cap \Sigma_{-i}^{\delta}\left(g^{*}\right)$. But, given that $g\left(s_{i}, \sigma_{-i}\right)=g^{*}-\ell_{1}\left(s_{i}\right)$ implies that $g\left(s_{i}^{\prime}, \sigma_{-i}\right)=g^{*}$, and given that $u_{i}\left(g^{*}\right)>u_{i}\left(g^{*}-\ell_{1}\left(s_{i}\right)\right)$, we can conclude that there exists $\delta>0$ such that $E u_{i}\left(s_{i}^{\prime}, \sigma_{-i}\right)>E u_{i}\left(s_{i}, \sigma_{-i}\right)$.

Case 2: Player $i$ cuts no links, but announces a missing link which harms her but benefits the other node. There does not exist $i j \in g^{*}$ for which $s_{i j}=0$. Assume that there exists $i j \notin g^{*}$ for which $s_{i j}=1, m u_{i}\left(g^{*}+i j, i j\right)<0$ and $m u_{j}\left(g^{*}+i j, i j\right)>0$.

Let $\ell_{2}\left(s_{i}\right)=\left\{i j \notin g^{*}: m u_{i}\left(g^{*}+i j, i j\right)<0, m u_{j}\left(g^{*}+i j, i j\right)>0, s_{i j}=1\right\}$. Clearly, $\ell_{2}\left(s_{i}\right) \neq \varnothing$.

Let $s_{i}^{\prime}$ be given by:

$$
s_{i j}^{\prime}= \begin{cases}s_{i j}, & \text { if } i j \notin g^{*} \text { and } m u_{i}\left(g^{*}+i j, i j\right)>0, \\ s_{i j}, & \text { if } i j \notin g^{*}, m u_{i}\left(g^{*}+i j, i j\right)<0 \quad \text { and } \quad m u_{j}\left(g^{*}+i j, i j\right)<0, \\ 0, & \text { if } i j \notin g^{*}, m u_{i}\left(g^{*}+i j, i j\right)<0 \quad \text { and } \quad m u_{j}\left(g^{*}+i j, i j\right)>0 \\ 1, & \text { if } i j \in g^{*}\end{cases}
$$

\footnotetext{
$\overline{28}$ With strategy $s_{i}$, player $i$ is cutting a link $i j \in g^{*}$ or announcing a new link $i j \notin g^{*}$ that gives a negative marginal payoff.
} 
Then, given that $\sigma_{-i} \in \Sigma_{-i}^{\delta}\left(g^{*}\right)$, there exists $\xi \in \mathbb{N}, \xi \neq 0$, such that:

$$
\frac{\operatorname{Pr}\left\{g\left(s_{i}, \sigma_{-i}\right)=g^{*}+\ell^{\prime}, \text { for some } \ell^{\prime} \subseteq \ell_{2}\left(s_{i}\right), \ell^{\prime} \neq \varnothing\right\}}{\operatorname{Pr}\left\{g\left(s_{i}, \sigma_{-i}\right) \neq g^{*}+\ell^{\prime}: \text { for all } \ell^{\prime} \subseteq \ell_{2}\left(s_{i}\right) \cap g\left(s_{i}, \sigma_{-i}\right), \ell^{\prime} \neq \varnothing\right\}} \geq 1-\xi \delta
$$

By definition of $s_{i}^{\prime}$, we know that $g\left(s_{i}, \sigma_{-i}\right)=g^{*}+\ell^{\prime}$ implies that $g\left(s_{i}^{\prime}, \sigma_{-i}\right)=g^{*}$ for all $\ell^{\prime} \subseteq \ell_{2}\left(s_{i}\right)$. Also, if $g\left(s_{i}, \sigma_{-i}\right)$ is such that there exists no $\ell^{\prime} \subseteq \ell_{2}\left(s_{i}\right) \cap$ $g\left(s_{i}, \sigma_{-i}\right), \ell^{\prime} \neq \varnothing$, then $g\left(s_{i}, \sigma_{-i}\right)=g\left(s_{i}^{\prime}, \sigma_{-i}\right)$. Due to $\beta$-strong supermodularity, we thus have $u_{i}\left(g^{*}+\ell^{\prime}\right)<u_{i}\left(g^{*}\right)$, for all $\ell^{\prime} \subseteq \ell_{2}\left(s_{i}\right), \ell^{\prime} \neq \varnothing$, implying that there exists $\delta>0$ such that $E u_{i}\left(s_{i}^{\prime}, \sigma_{-i}\right)>E u_{i}\left(s_{i}, \sigma_{-i}\right)$.

Case 3: Player $i$ cuts no links, but announces a missing link which harms her and the other node. Assume now that neither the conditions enumerated in Case 1 nor in Case 2 hold. Suppose $s_{i}$ is such that there exists $i j \notin g^{*}$ for which $s_{i j}=$ $1, m u_{i}\left(g^{*}+i j, i j\right)<0$ and $m u_{j}\left(g^{*}+i j, i j\right)<0$. Let $\ell_{3}\left(s_{i}\right)=\left\{i j \notin g^{*}:\right.$ $\left.m u_{i}\left(g^{*}+i j, i j\right)<0, m u_{j}\left(g^{*}+i j, i j\right)<0, s_{i j}=1\right\}$. By assumption, $\ell_{3}\left(s_{i}\right) \neq \varnothing$. Let $s_{i}^{\prime}$ be given by:

$$
s_{i j}^{\prime}= \begin{cases}s_{i j}, & \text { if } m u_{i}\left(g^{*}+i j, i j\right)>0 \\ 0, & \text { if } m u_{i}\left(g^{*}+i j, i j\right)<0 \\ 1, & \text { if } i j \in g^{*}\end{cases}
$$

Then, given that $\sigma_{-i} \in \Sigma_{-i}^{\delta}\left(g^{*}\right)$, there exists some number $\xi \in \mathbb{N}, \xi \neq 0$, such that:

$$
\frac{\operatorname{Pr}\left\{g\left(s_{i}, \sigma_{-i}\right)=g^{*}+i j, \text { for some } i j \in \ell_{3}\left(s_{i}\right)\right\}}{\operatorname{Pr}\left\{g\left(s_{i}, \sigma_{-i}\right) \neq g^{*}+i j: \text { for all } i j \in \ell_{3}\left(s_{i}\right) \cap g\left(s_{i}, \sigma_{-i}\right)\right\}} \geq \min \left\{1-\xi \delta, \frac{1}{\delta}\right\} .
$$

By definition of $s_{i}^{\prime}$, we know that $g\left(s_{i}, \sigma_{-i}\right)=g^{*}+i j$ implies that $g\left(s_{i}^{\prime}, \sigma_{-i}\right)=g^{*}$. Also, if $g\left(s_{i}, \sigma_{-i}\right)$ is such that there exists no $i j \in g\left(s_{i}, \sigma_{-i}\right)$, then $g\left(s_{i}, \sigma_{-i}\right)=$ $g\left(s_{i}^{\prime}, \sigma_{-i}\right)$. By construction, we thus have $u_{i}\left(g^{*}+i j\right)<u_{i}\left(g^{*}\right)$, implying that there exists $\delta>0$ such that $E u_{i}\left(s_{i}^{\prime}, \sigma_{-i}\right)>E u_{i}\left(s_{i}, \sigma_{-i}\right)$.

Lemma 3 Let $g^{*} \in P N E(u)$ and $\sigma_{-i} \in \widetilde{\Sigma}_{-i}^{\epsilon} \cap \Sigma_{-i}^{\delta}\left(g^{*}\right)$. Suppose that, for some $s_{i} \in$ $S_{i}$, there exists $i j \notin g^{*}, i j \in v_{i}(g, u)$ such that $s_{i j}=1$, and there exists some $k \neq j$ such that either $i k \in g^{*}$ and $s_{i k}=0$, or $i k \notin g^{*}, m u_{i}\left(g^{*}+i k, i k\right)<0$ and $s_{i k}=1$, or both. Then, there exists a $\delta>0$ such that $E u_{i}\left(s_{i}, \sigma_{-i}\right)<E u_{i}\left(s_{i}^{0}\left(g^{*}, s_{i}, i j\right), \sigma_{-i}\right)$.

Proof of Lemma 3 Let $s_{i}$ and $s_{i}^{0}\left(g, s_{i}, i j\right)$ defined as in Lemma 2. Again, we distinguish three cases.

Case 1: Player $i$ cuts a link. There exists $j \in N$ such that $i j \in g^{*}$ and $s_{i j}=0$. Let then $\ell_{1}\left(s_{i}\right)=\left\{i j \in g^{*}: s_{i j}=0\right\}$. Clearly, $\ell_{1}\left(s_{i}\right) \neq \varnothing$. For all $\sigma_{-i} \in \widetilde{\Sigma}_{-i}^{\epsilon} \cap \Sigma_{-i}^{\delta}\left(g^{*}\right)$, there exists $\xi \in \mathbb{N}, \xi \neq 0$, such that $\operatorname{Pr}\left\{g\left(s_{i}, \sigma_{-i}\right)=g^{*}-\ell_{1}\left(s_{i}\right)\right\} \geq 1-\xi \delta$. But, given that $g\left(s_{i}, \sigma_{-i}\right)=g^{*}-\ell_{1}\left(s_{i}\right)$ implies that $g\left(s_{i}^{0}\left(g^{*}, s_{i}, i j\right), \sigma_{-i}\right)=g^{*}$, and given that $u_{i}\left(g^{*}\right)>u_{i}\left(g^{*}-\ell_{1}\left(s_{i}\right)\right)$, we conclude that there exists $\delta>0$ such that $E u_{i}\left(s_{i}^{0}\left(g^{*}, s_{i}, i j\right), \sigma_{-i}\right)>E u_{i}\left(s_{i}, \sigma_{-i}\right)$. 
Case 2: Player $i$ cuts no links, but announces a missing link which harms her but benefits the other node. $s_{i} \in S_{i}$ is such that there exists no $j \in N$ such that $i j \in g^{*}$ and $s_{i j}=0$. But, there exists $i j \notin g^{*}$ for which $s_{i j}=1, m u_{i}\left(g^{*}+i j, i j\right)<$ 0 and $m u_{j}\left(g^{*}+i j, i j\right)>0$. Define $\ell_{2}\left(s_{i}\right)=\left\{i j \notin g^{*}: m u_{i}\left(g^{*}+i j, i j\right)<\right.$ $\left.0, m u_{j}\left(g^{*}+i j, i j\right)>0, s_{i j}=1\right\}$. Clearly, $\ell_{2}\left(s_{i}\right) \neq \varnothing$. Then, given that $\sigma_{-i} \in$ $\Sigma_{-i}^{\delta}\left(g^{*}\right)$, there exists $\xi \in \mathbb{N}, \xi \neq 0$, such that:

$\frac{\operatorname{Pr}\left\{g\left(s_{i}, \sigma_{-i}\right)=g^{*}+\ell^{\prime}, \text { for some } \ell^{\prime} \subseteq \ell_{2}\left(s_{i}\right), \ell^{\prime} \neq \varnothing\right\}}{\operatorname{Pr}\left\{g\left(s_{i}, \sigma_{-i}\right) \neq g^{*}+\ell^{\prime}: \text { for all } \ell^{\prime} \subseteq \ell_{2}\left(s_{i}\right) \cap g\left(s_{i}, \sigma_{-i}\right), \ell^{\prime} \neq \varnothing\right\}} \geq(1-\xi \delta)\left(1-\delta^{n}\right)$.

We know that $g\left(s_{i}, \sigma_{-i}\right)=g^{*}+\ell^{\prime}$ implies that $g\left(s_{i}^{0}\left(g^{*}, s_{i}, i j\right), \sigma_{-i}\right)=g^{*}$, for all $\ell^{\prime} \subseteq \ell_{2}\left(s_{i}\right)$. Also, if $g\left(s_{i}, \sigma_{-i}\right)$ is such that there exists no $\ell^{\prime} \subseteq \ell_{2}\left(s_{i}\right) \cap$ $g\left(s_{i}, \sigma_{-i}\right), \ell^{\prime} \neq \varnothing$, then $g\left(s_{i}^{0}\left(g^{*}, s_{i}, i j\right), \sigma_{-i}\right)=g\left(s_{i}, \sigma_{-i}\right)$. Due to $\beta$-strong supermodularity, we thus have $u_{i}\left(g^{*}+\ell^{\prime}\right)<u_{i}\left(g^{*}\right)$, for all $\ell^{\prime} \subseteq \ell_{2}\left(s_{i}\right), \ell^{\prime} \neq \varnothing$, implying that there exists $\delta>0$ such that $E u_{i}\left(s_{i}^{0}\left(g^{*}, s_{i}, i j\right), \sigma_{-i}\right)>E u_{i}\left(s_{i}, \sigma_{-i}\right)$.

Case 3: Player $i$ cuts no links, but announces a missing link which harms her and the other node. Assume now that neither the conditions enumerated in Case 1 nor in Case 2 hold. Suppose $s_{i}$ is such that there exists some $i j \notin g^{*}$ for which $s_{i j}=1$, $m u_{i}\left(g^{*}+i j, i j\right)<0$ and $m u_{j}\left(g^{*}+i j, i j\right)<0$. Define now $\ell_{3}\left(s_{i}\right)=\left\{i j \notin g^{*}:\right.$ $\left.m u_{i}\left(g^{*}+i j, i j\right)<0, m u_{j}\left(g^{*}+i j, i j\right)<0, s_{i j}=1\right\}$. By assumption, $\ell_{3}\left(s_{i}\right) \neq \varnothing$. Let $s_{i}^{\prime}$ be given by:

$$
s_{i j}^{\prime}= \begin{cases}s_{i j}, & \text { if } m u_{i}\left(g^{*}+i j, i j\right)>0 \\ 0, & \text { if } m u_{i}\left(g^{*}+i j, i j\right)<0 \\ 1, & \text { if } i j \in g^{*}\end{cases}
$$

Then, given that $\sigma_{-i} \in \Sigma_{-i}^{\delta}\left(g^{*}\right)$, there exists $\xi \in \mathbb{N}, \xi \neq 0$, such that:

$$
\frac{\operatorname{Pr}\left\{g\left(s_{i}, \sigma_{-i}\right)=g^{*}+i j \text {, for some } i j \in \ell_{3}\left(s_{i}\right)\right\}}{\operatorname{Pr}\left\{g\left(s_{i}, \sigma_{-i}\right) \neq g^{*}+i j: \text { for all } i j \in \ell_{3}\left(s_{i}\right) \cap g\left(s_{i}, \sigma_{-i}\right)\right\}} \geq \min \left\{1-\xi \delta, \frac{1}{\delta}\right\} .
$$

By definition of $s_{i}^{\prime}$, we know that $g\left(s_{i}, \sigma_{-i}\right)=g^{*}+i j$ implies that $g\left(s_{i}^{0}\left(g^{*}, s_{i}, i j\right), \sigma_{-i}\right)=g^{*}$. Also, if $g\left(s_{i}, \sigma_{-i}\right)$ is such that there exists no $i j \in$ $\ell_{3}\left(s_{i}\right) \cap g\left(s_{i}, \sigma_{-i}\right)$, then $g\left(s_{i}, \sigma_{-i}\right)=g\left(s_{i}^{0}\left(g^{*}, s_{i}, i j\right), \sigma_{-i}\right)$. By construction, we have $u_{i}\left(g^{*}+i j\right)<u_{i}\left(g^{*}\right)$, implying that there exists $\delta>0$ such that $E u_{i}\left(s_{i}^{0}\left(g^{*}, s_{i}\right.\right.$, $\left.i j), \sigma_{-i}\right)>E u_{i}\left(s_{i}, \sigma_{-i}\right)$.

We are now ready to establish Proposition 3.

By Lemmas 2 and 3, there exists $\delta>0$ such that $\widehat{\sigma}_{i}^{\sigma_{-i}} \in \widetilde{\Sigma}_{i}^{\epsilon} \cap \Sigma_{i}^{\delta}\left(g^{*}\right)$. By Kakutani's fixed point theorem, $\rho_{i}^{g^{*}}$ is non-empty and has a fixed point, which is an $\epsilon$-proper equilibrium. We take a sequence of $\left\{\epsilon_{t}\right\}_{t \in \mathbb{N}}$ such that $\widetilde{\Sigma}_{i}^{\epsilon_{t}} \cap \Sigma^{\delta}\left(g^{*}\right) \rightarrow$ $\Sigma^{\delta}\left(g^{*}\right)$. The corresponding sequence of $\epsilon_{t}$-proper equilibria converges to a proper equilibrium $\sigma^{*}$. By Lemma 2, $g\left(\sigma^{*}\right)=g^{*}$, and thus $g^{*} \in P R E(u)$. 
Open Access This article is distributed under the terms of the Creative Commons Attribution Noncommercial License which permits any noncommercial use, distribution, and reproduction in any medium, provided the original author(s) and source are credited.

\section{Appendix}

Proof of Claim 1. Let $i j \in g$. First, note that for all $k \in N, d_{i k}(g) \leq d_{i k}(g-i j)$. Define:

$$
\chi(g, i j)=\left\{k \in N: d_{i k}(g)<d_{i k}(g-i j)\right\} .
$$

In particular, $j \in \chi(g, i j)$. Then,

$$
m u_{i}(g, i j)=\sum_{k \in \chi(g, i j)}\left[\delta^{d_{i k}(g)}-\delta^{d_{i k}(g-i j)}\right]-c .
$$

For $l \in N, l \neq j$, let $i l \in g$. Define $\chi(g, i j, i l)=\left\{k \in N: d_{i k}(g)<d_{i k}(g-i j-\right.$ il)\}. Then,

$$
m u_{i}(g, i j+i l)=\sum_{k \in \chi(g, i j, i l)}\left[\delta^{d_{i k}(g)}-\delta^{d_{i k}(g-i j-i l)}\right]-2 c .
$$

Note the following.

First, $\chi(g, i j) \cup \chi(g, i l) \subset \chi(g, i j, i l)$. Indeed, for all $k \in \chi(g, i j)$, we have $d_{i k}(g)<d_{i k}(g-i j) \leq d_{i k}(g-i j-i l)$. Idem for $\chi(g, i l)$.

Second, $\chi(g, i j) \cap \chi(g, i l)=\emptyset$. Indeed, let $k \in \chi(g, i j) \cap \chi(g, i l)$. Then, both $l$ and $j$ are on (two different) shortest paths in $g$ between $i$ and $k$. Consider the shortest path in $g$ between $i$ and $k$ that crosses through $l$. This path still exists in $g-i j$, implying that $d_{i k}(g)=d_{i k}(g-i j)$, in contradiction with $k \in \chi(g, i l)$.

Third, $\delta^{d_{i k}(g)}-\delta^{d_{i k}(g-i j-i l)} \geq \delta^{d_{i k}(g)}-\delta^{d_{i k}(g-i j)}$, as $d_{i k}(g-i j) \leq d_{i k}(g-i j-i l)$. Similarly for $\delta^{d_{i k}(g)}-\delta^{d_{i k}(g-i l)}$.

Altogether, this implies that $m u_{i}(g, i j+i l) \geq m u_{i}(g, i j)+m u_{i}(g, i l)$.

Proof of Claim 2. Let $g \in P S(u)$ for the co-author model. Then, $g$ is formed of internally completely connected components (Jackson and Wolinsky 1996). Let $i \in N$ and consider the component of $g$ to which $i$ belongs. If $n_{i}(g)$ is the number of $i$ 's direct contacts in the network, then the size of this component is $n_{i}(g)+1$. To simplify notation, we write $n_{i}$ instead of $n_{i}(g)$. Assume that $i$ is not isolated, so that $n_{i} \geq 2$. Then,

$$
m u_{i}(g, i j)=\frac{1}{n_{i}}, \quad \text { for all } i j \in g .
$$

Therefore, given some $\ell \subseteq L_{i}(g)$, we have:

$$
\sum_{i j \in \ell} m u_{i}(g, i j)=\frac{|\ell|}{n_{i}}
$$


Instead,

$$
m u_{i}(g, \ell)=\frac{n_{i}-|\ell|+1}{n_{i}-|\ell|} \frac{|\ell|}{n_{i}}-\frac{|\ell|}{n_{i}\left(n_{i}-|\ell|\right)}=\frac{|\ell|}{n_{i}} .
$$

Proof of Claim 3. First, note that for the information transmission network payoffs we have:

$$
m u_{i}(g, \ell)=\left[1-\prod_{j: i j \in \ell} p_{j i}\right] \prod_{j: i j \in g \backslash \ell} p_{j i}-c|\ell|, \quad \text { for all } \ell \in L_{i}(g)
$$

Therefore, establishing that

$$
m u_{i}(g, \ell) \geq \sum_{i j \in \ell} m u_{i}(g, i j)
$$

is equivalent to showing that

$$
1-\prod_{j: i j \in \ell} p_{j i} \geq \sum_{j: i j \in \ell}\left[1-p_{j i}\right] \prod_{k: i k \in \ell, k \neq j} p_{k i}
$$

This last inequality derives from the following result.

Remark Let $1 \geq x_{i} \geq 0, i \in\{1, \ldots, m\}$. Then, $1-\prod_{i \in\{1, \ldots m\}} x_{i} \geq \sum_{i=1}^{m}\left(1-x_{i}\right)$ $\prod_{j \in\{1, \ldots, m\}, j \neq i} x_{j}$.

A proof by induction is the following. First, the inequality is trivially satisfied when $m=2$. Suppose that it holds for some $m \geq 2$. We show that it still holds at $m+1$, and the induction will follow. Let $x_{1}^{\prime}=\prod_{i \in\{1, \ldots m\}} x_{i}$ and $x_{2}^{\prime}=x_{m+1}$. Clearly, $0, \leq x_{1}^{\prime}, x_{2}^{\prime} \leq 1$. The inequality applied to these two values gives

$$
1-\left(\prod_{i \in\{1, \ldots m\}} x_{i}\right) x_{m+1} \geq\left(1-\prod_{i \in\{1, \ldots m\}} x_{i}\right) x_{m+1}+\left(1-x_{m+1}\right) \prod_{i \in\{1, \ldots m\}} x_{i}
$$

The result then follows by induction on $m$.

Example (violation of strong link responsiveness) We provide an example where strong link responsiveness is violated and there exists a proper equilibrium network which is not pairwise-stable. This will show that the strong link-responsiveness is necessary to guarantee that proper equilibrium networks are pairwise-stable.

Let $N=\{1,2,3,4\}$. The strategy set of a player $i$ is $S_{i}=\{$ for $j<k<l \in$ $\left.N \backslash\{i\},\left(s_{i j}, s_{i k}, s_{i l}\right) \in\{0,1\}^{3}\right\}$. The players are labeled as below: 


\section{Player $1 \quad$ Player 2}

\section{Player 3 Player 4}

In the following networks the payoff of each player is given next to the corresponding node.
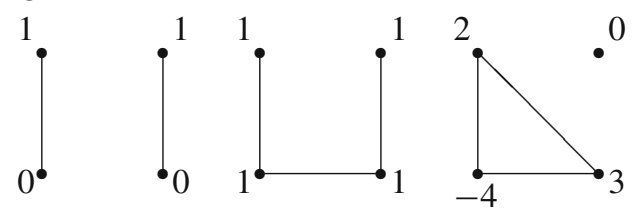

$g_{0}$

$g_{1}$

$g_{2}$

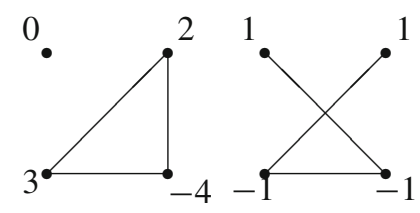

$g_{3}$

$g_{4}$

Observe that $g_{0}$ is not pairwise-stable as players 3 and 4 can form a link which benefits both.
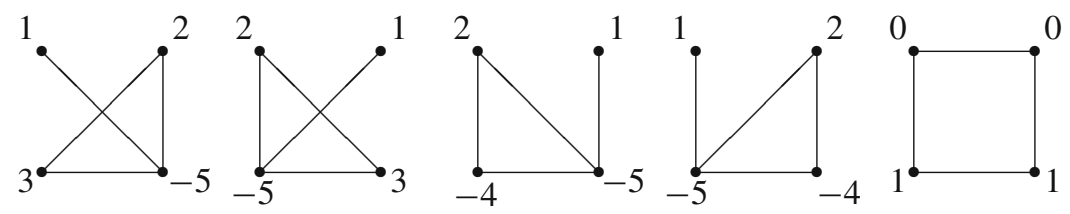

We will construct the example such that player 1's and player 2's payoffs are symmetric. In visual terms if two networks are mirror images of each other then player 1's payoff in one equals player 2's payoff in the other. Such a symmetry will also hold between player 3 and player 4 . This construction will make the example simpler and decrease the computational burden.
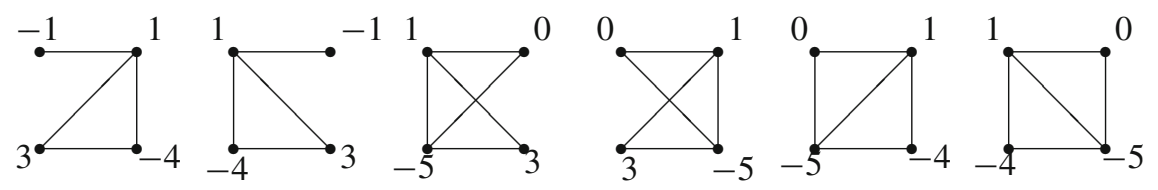

For the rest of the networks each player's payoff will depend only on her links. Given a network $g$ and two players $i$ and $j$, let $g_{i j}$ be a binary valued function such that

$$
g_{i j}= \begin{cases}0, & \text { if } i j \notin g \\ 1, & \text { if } i j \in g .\end{cases}
$$

Given a network $g$, the payoffs of players 1 and 2 are:

$$
\begin{aligned}
& u_{1}(g)=g_{13}+g_{14}-g_{12}, \\
& u_{2}(g)=g_{23}+g_{24}-g_{12}
\end{aligned}
$$


The payoffs of players 3 and 4 are

$$
\begin{aligned}
& u_{3}(g)= \begin{cases}0, & \text { if } L_{3}(g)=\{13\} \text { or } L_{3}(g)=\{23\}, \\
-2, & \text { if } L_{3}(g)=\{13,23\} \text { or } L_{3}(g)=\{34\}, \\
-5, & \text { if } L_{3}(g)=\{13,23,34\} \\
1, & \text { otherwise, }\end{cases} \\
& u_{4}(g)= \begin{cases}0, & \text { if } L_{4}(g)=\{14\} \text { or } L_{3}(g)=\{24\}, \\
-2, & \text { if } L_{4}(g)=\{14,24\} \text { or } L_{4}(g)=\{34\}, \\
-5, & \text { if } L_{4}(g)=\{14,24,34\}, \\
1, & \text { otherwise. }\end{cases}
\end{aligned}
$$

The above payoff functions violate strong link responsiveness, because player 1 is indifferent between having a link with player 3 or having a link with player 4 . But the weak link responsiveness is satisfied as the deletion of a single link always affects the payoffs of the involved parties.

The strategy profile $s^{*}=\left(s_{1}^{*}, s_{2}^{*}, s_{3}^{*}, s_{4}^{*}\right)$ such that,

$$
s_{1}^{*}=(0,1,1), \quad s_{2}^{*}=(0,1,1), \quad s_{3}^{*}=(1,0,0), \quad s_{4}^{*}=(0,1,0)
$$

leads to $g_{0}$. We will show that $s^{*}$ is a proper equilibrium, although $g_{0}$ is not pairwisestable.

The $\varepsilon$-proper equilibrium $\sigma^{\varepsilon}$ that converges to $s^{*}$ is

$$
\begin{gathered}
\sigma_{1}^{\varepsilon}((1,1,1))=\varepsilon^{10}, \sigma_{1}^{\varepsilon}((0,1,0))=\varepsilon^{11}, \sigma_{1}^{\varepsilon}((1,1,0))=\varepsilon^{12}, \sigma_{1}^{\varepsilon}((0,0,1))=\varepsilon^{13}, \\
\sigma_{1}^{\varepsilon}((1,0,1))=\varepsilon^{14}, \sigma_{1}^{\varepsilon}((0,0,0))=\varepsilon^{15}, \sigma_{1}^{\varepsilon}((1,0,0))=\varepsilon^{16}, \sigma_{1}^{\varepsilon}((0,1,1))=1-\sum_{i=10}^{i=16} \varepsilon^{i} \\
\sigma_{2}^{\varepsilon}((1,1,1))=\varepsilon^{10}, \sigma_{2}^{\varepsilon}((0,0,1))=\varepsilon^{11}, \sigma_{2}^{\varepsilon}((1,0,1))=\varepsilon^{12}, \sigma_{2}^{\varepsilon}((0,1,0))=\varepsilon^{13}, \\
\sigma_{2}^{\varepsilon}((1,1,0))=\varepsilon^{14}, \sigma_{2}^{\varepsilon}((0,0,0))=\varepsilon^{15}, \sigma_{2}^{\varepsilon}((1,0,0))=\varepsilon^{16}, \sigma_{2}^{\varepsilon}((0,1,1))=1-\sum_{i=10}^{i=16} \varepsilon^{i} \\
\sigma_{3}^{\varepsilon}((0,1,0))=\varepsilon, \sigma_{3}^{\varepsilon}((0,1,1))=\varepsilon^{2}, \sigma_{3}^{\varepsilon}((1,0,1))=\varepsilon^{3}, \sigma_{3}^{\varepsilon}((0,0,0))=\varepsilon^{4}, \\
\sigma_{3}^{\varepsilon}((0,0,1))=\varepsilon^{5}, \sigma_{3}^{\varepsilon}((1,1,0))=\varepsilon^{6}, \sigma_{3}^{\varepsilon}((1,1,1))=\varepsilon^{7}, \sigma_{3}^{\varepsilon}((1,0,0))=1-\sum_{i=1}^{i=7} \varepsilon^{i} \\
\sigma_{4}^{\varepsilon}((1,0,0))=\varepsilon, \sigma_{4}^{\varepsilon}((1,0,1))=\varepsilon^{2}, \sigma_{4}^{\varepsilon}((0,1,1))=\varepsilon^{3}, \sigma_{4}^{\varepsilon}((0,0,0))=\varepsilon^{4}, \\
\sigma_{4}^{\varepsilon}((0,0,1))=\varepsilon^{5}, \sigma_{4}^{\varepsilon}((1,1,0))=\varepsilon^{6}, \sigma_{4}^{\varepsilon}((1,1,1))=\varepsilon^{7}, \sigma_{4}^{\varepsilon}((0,1,0))=1-\sum_{i=1}^{i=7} \varepsilon^{i}
\end{gathered}
$$

As players 1 and 2 receive a payoff of 1 for each link they make with players 3 or 4 and incur a loss of 1 if they form a link among each other $s_{1}=(0,1,1)$ and $s_{2}=(0,1,1)$ are dominant strategies for players 1 and 2 , respectively. 
For player 3 , it is clear that the only strategy that might be a better response to $\sigma_{-3}^{\varepsilon}$ than $s_{3}^{*}$ is $s_{3}^{\prime}=(1,0,1)$. We will show that for player 3 and $s_{3}^{\prime}$,

$$
E u_{3}\left(s_{3}^{*}, \sigma_{-3}^{\varepsilon}\right)>E u_{3}\left(s_{3}^{\prime}, \sigma_{-3}^{\varepsilon}\right)
$$

Observe that the mistakes of players 1 and 2 have probabilities of lower orders than mistakes of player 4 . Hence while comparing $E u_{3}\left(s_{3}^{*}, \sigma_{-3}^{\varepsilon}\right)$ with $E u_{3}\left(s_{3}^{\prime}, \sigma_{-3}^{\varepsilon}\right), \sigma_{4}^{\varepsilon}$ is decisive and the mistakes of players 1 and 2 can be ignored.

$$
E u_{3}\left(s_{1}^{*}, s_{2}^{*}, s_{3}^{*}, \sigma_{4}^{\varepsilon}\right)>E u_{3}\left(s_{1}^{*}, s_{2}^{*}, s_{3}^{\prime}, \sigma_{4}^{\varepsilon}\right) \Longrightarrow E u_{3}\left(s_{3}^{*}, \sigma_{-3}^{\varepsilon}\right)>E u_{3}\left(s_{3}^{\prime}, \sigma_{-3}^{\varepsilon}\right) .
$$

A direct calculation shows that

$$
E u_{3}\left(s_{1}^{*}, s_{2}^{*}, s_{3}^{*}, \sigma_{4}^{\varepsilon}\right)=0 \quad \text { and } E u_{3}\left(s_{1}^{*}, s_{2}^{*}, s_{3}^{\prime}, \sigma_{4}^{\varepsilon}\right)=-4 \varepsilon^{2}+\varepsilon^{3}-\varepsilon^{5}-4 \varepsilon^{7}<0
$$

Then $s_{3}^{*}$ is the unique best response to $\sigma_{-3}^{\varepsilon}$. Due to the symmetry between players 3 and 4 , it holds that for $s_{4}^{\prime}=(0,1,1)$

$$
E u_{4}\left(s_{4}^{*}, \sigma_{-4}^{\varepsilon}\right)>E u_{4}\left(s_{4}^{\prime}, \sigma_{-4}^{\varepsilon}\right) .
$$

Then $s^{*}$ is a proper equilibrium, ${ }^{29}$ although the network it supports, $g_{0}$, is not pairwise-stable.

Example (violation of $\beta$-strong supermodularity) We provide an example where a pairwise-Nash network that violates $\beta$-strong supermodularity can not be supported by a proper equilibrium. Let $N=\{1,2,3\}$ and consider the network payoffs defined below:
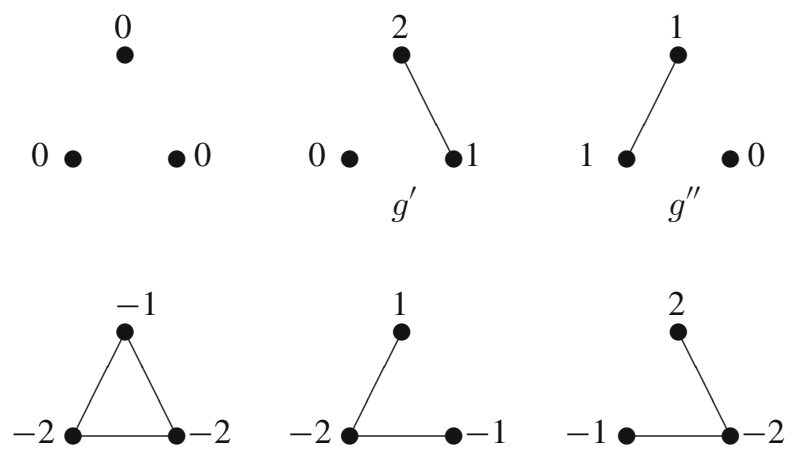
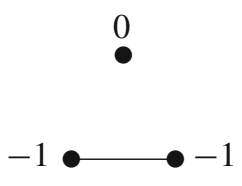

$g^{\prime \prime}$
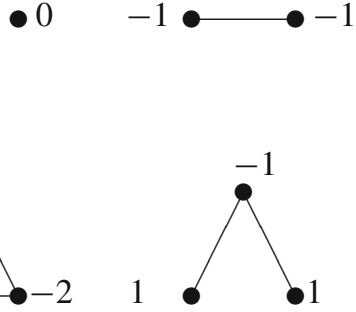

${ }_{29}$ It is straightforward to check that for arbitrarily small $\varepsilon$ the completely mixed strategy profile $\sigma^{\varepsilon}$ satisfies $\forall i \in N, \forall s_{i}, s_{i}^{\prime} \in S_{i}, E u_{i}\left(s_{i}, \sigma_{-i}^{\varepsilon t}\right)>E u_{i}\left(s_{i}^{\prime}, \sigma_{-i}^{\varepsilon}\right)$ implies that $\sigma_{i}^{\varepsilon}\left(s_{i}^{\prime}\right) \leq \varepsilon \cdot \sigma_{i}^{\varepsilon}\left(s_{i}\right)$.

We omit the calculations. 
Denote by 1 the player at the top, and by 2 and 3 the players at the bottom, and let $S_{1}=\left\{\left(s_{12}, s_{13}\right) \in\{0,1\}^{2}\right\}, S_{2}=\left\{\left(s_{21}, s_{23}\right) \in\{0,1\}^{2}\right\}$ and $S_{3}=\left\{\left(s_{31}, s_{32}\right) \in\right.$ $\left.\{0,1\}^{2}\right\}$.

For players 2 and 3 it is a dominant strategy to announce a single link with player 1.

The only two pairwise-Nash equilibrium networks are $g^{\prime}$ and $g^{\prime \prime} \cdot{ }^{30}$ But $g^{\prime \prime}$ does not satisfy $\beta$-strong supermodularity, because

$$
u_{1}\left(g^{\prime \prime}+13-12\right)-u_{1}\left(g^{\prime \prime}\right)=1>-1=m u_{1}\left(g^{\prime \prime}+13\right) \text {. }
$$

In all Nash equilibria that support $g^{\prime \prime}$, player 3 never announces a link with player 1 . Hence, $g^{\prime \prime}$ can not be supported by a pure strategy proper equilibrium.

\section{References}

Bala V, Goyal S (2000) A noncooperative model of network formation. Econometrica 68:1181-1230

Bloch F, Jackson MO (2006) Definitions of equilibrium in network formation games. Int J Game Theory 34:305-318

Bloch F, Jackson MO (2007) The formation of networks with transfers among players. J Econ Theory 133:83-110

Calvó-Armengol A (2004) Job contact networks. J Econ Theory 115:191-206

Dutta B, Tijs S, Nouweland Avan den (1998) Link formation in cooperative situations. Int J Game Theory 27:245-256

Dutta B, Mutuswami S (1997) Stable networks. J Econ Theory 76:322-344

Fréchet M (1951) Sur les tableaux de corrélation dont les marges sont données. Ann l'Université Lyon 14:53-77

Gilles R, Sarangi S (2005) Stable Networks and Convex Payoffs, Departmental Working Papers 2005-13, Department of Economics, Louisiana State University

Goyal S, Joshi S (2006) Unequal Connections. Int J Game Theory 34:319-349

Jackson MO (2004) A survey of models of network formation: stability and efficiency, 2004 In: Demange G, Wooders M (eds) Group Formation in Economics; Networks, Clubs and Coalitions, Chapter 1. Cambridge University Press, Cambridge

Jackson MO, Rogers B (2006) Search in the formation of large networks: How random are socially generated networks?. Am Econ Rev (2006, forthcoming)

Jackson MO, Wolinsky A (1996) A strategic model of social and economic networks. J Econ Theory 71: 44-74

Jackson MO, Watts A (2001) The existence of pairwise stable networks. Seoul J Econ 14:299-321

Jackson MO, Watts A (2002) The evolution of social and economic networks. J Econ Theory 106:265-295

Jackson MO, Nouweland Avan den (2005) Strongly stable networks. Games Econ Behav 51(2):420-444

Mailath GJ, Samuelson L, Swinkels JM (1997) How proper is sequential equilibrium?. Games Econ Behav 18:193-218

Myerson RB (1978) Refinements of the Nash equilibrium concept. Int J Game Theory 7:73-80

Myerson RB (1991) Game theory: analysis of conflict. Harvard University Press, Cambridge

Selten R (1975) Rexamination of the perfection concept for equilibrium points in extensive games. Int J Game Theory 4:25-55

$30 s^{\prime}=\left(s_{1}^{\prime}, s_{2}^{\prime}, s_{3}^{\prime}\right)=((0,1),(1,0),(1,0))$ is a Nash equilibrium that supports $g^{\prime}$. There are 5 more Nash equilibria that support $g^{\prime}$.

$g^{\prime \prime}$ is a Nash equilibrium network for the following Nash equilibrium pure strategy profiles $s=\left(s_{1}, s_{2}, s_{2}\right)$ : $((1,0),(1,0),(0,0)),((1,0),(1,1),(0,0)),((1,0),(1,0),(0,1))$. 
van Damme E (1984) A relation between perfect equilibria in extensive form games and proper equilibria in normal form games. Int J Game Theory 13:1-13

Yamamoto Y (1993) A path-following procedure to find a proper equilibrium of finite games. Int J Game Theory 22:249-259 